\title{
MIMO-SAR: Opportunities and Pitfalls
}

\author{
Gerhard Krieger, Fellow, IEEE
}

\begin{abstract}
This paper reviews advanced radar architectures that employ multiple transmit and multiple receive antennas to improve the performance of future synthetic aperture radar (SAR) systems. These advanced architectures have been dubbed multiple-input multiple-output SAR (MIMO-SAR) in analogy to MIMO communication systems. Considerable confusion arose, however, with regard to the selection of suitable waveforms for the simultaneous transmission via multiple channels. It is shown that the mere use of orthogonal waveforms is insufficient for the desired performance improvement in view of most SAR applications. As a solution to this fundamental MIMO-SAR challenge, a new class of short-term shift-orthogonal waveforms is introduced. The short-term shift orthogonality avoids mutual interferences from the radar echoes of closely spaced scatterers, while interferences from more distant scatterers are suppressed by digital beamforming on receive in elevation. Further insights can be gained by considering the data acquisition of a side-looking imaging radar in a 3-D information cube. It becomes evident that the suggested waveforms fill different subspaces that can be individually accessed by a multichannel receiver. For completeness, the new class of short-term shift-orthogonal waveforms is also compared to a recently proposed pair of orthogonal frequency-division multiplexing waveforms. It is shown that both sets of waveforms require essentially the same principle of range time to elevation angle conversion via a multichannel receiver in order to be applicable for MIMO-SAR imaging without interference.
\end{abstract}

Index Terms-Digital beamforming (DBF), multidimensional waveform encoding, multiple-input multiple-output synthetic aperture radar (MIMO-SAR), orthogonal frequency-division multiplexing (OFDM).

\section{INTRODUCTION}

$\mathbf{S}$ YNTHETIC aperture radar (SAR) is a powerful remote sensing technique that can provide high-resolution radar images of the terrestrial surface independent of weather and sunlight illumination. This capability makes SAR an ideal candidate for a global Earth observatory that continuously monitors the plethora of natural and anthropogenic processes which permanently restructure the surface of our planet. The mapping capabilities of current SAR sensors are, however, insufficient to reach this ambitious goal with a reasonable number of satellites. Next-generation SAR missions like Tandem-L will employ advanced digital beamforming (DBF) techniques to improve the imaging performance by more than one order of magnitude if compared to state-of-the-art sensors like TerraSAR-X [1]. In the future, further improvements can be achieved by combining DBF on receive with multiple transmit channels ra-

Manuscript received August 11, 2012; revised January 3, 2013 and April 18, 2013; accepted May 14, 2013. Date of publication June 28, 2013; date of current version February 27, 2014.

The author is with the Microwaves and Radar Institute, German Aerospace Center (DLR), 82234 Weßling, Germany (e-mail: gerhard.krieger@dlr.de).

Color versions of one or more of the figures in this paper are available online at http://ieeexplore.iee.org.

Digital Object Identifier 10.1109/TGRS.2013.2263934 diating appropriately designed waveforms [2]. These enhanced architectures, now dubbed multiple-input multiple-output SAR (MIMO-SAR), offer not only the opportunity to map wider image swaths with improved spatial resolution, but they also enable novel SAR imaging modes that are well suited to mitigate or even resolve hitherto contradicting user requirements.

This paper provides a critical review of recently suggested MIMO-SAR concepts and discusses their validity and pitfalls in the context of wide-swath SAR imaging. For this, Section II provides a short summary of the early developments toward MIMO radar, together with the manifold opportunities that have been suggested over the last years in the special context of SAR imaging. Here, an important issue arises with regard to the separation of the radar echoes that arise from the simultaneous scene illumination by multiple transmit waveforms. To this end, several types of orthogonal signals have been suggested in the literature, and Section III provides a critical analysis of the suitability of such waveforms in the context of wide-swath SAR imaging. To overcome some fundamental issues with regard to these previously suggested orthogonal signals, Section IV introduces the novel concept of shortterm shift-orthogonal waveforms. It is shown that the usage of these particular waveforms enables, in combination with DBF on receive, a MIMO-SAR imaging system that avoids the performance degradations associated with the use of the conventional, hitherto-proposed orthogonal signals, as derived in Section III. To further explain the intimate connection between MIMO-SAR imaging, short-term shift orthogonality, and DBF on receive, Section V introduces the visual concept of the information cube, which provides further insights into the validity and pitfalls of the MIMO-SAR data collection process. Recently, it has been suggested that a special class of orthogonal frequency-division multiplexing (OFDM) waveforms is also suitable for MIMO-SAR imaging. Section VI discusses this class of waveforms and shows their similarities and differences if compared to the short-term shift-orthogonal waveforms in the context of MIMO-SAR imaging. This paper concludes with a short outlook on advanced MIMO-SAR modes in Section VII and a final summary in Section VIII.

\section{EARly DEVElopments TOWARD MIMO-SAR}

A radar receiver with $N_{\mathrm{Rx}}$ apertures allows a simultaneous sampling of the arriving wavefronts with $N_{\mathrm{Rx}}$ effective phase centers. The number of effective phase centers can be significantly increased by using not only one but multiple transmitters. If $N_{\mathrm{Tx}}$ transmitters are employed in addition to $N_{\mathrm{Rx}}$ receivers, the maximum number of possible phase centers increases from $N_{\mathrm{DBF}}=N_{\mathrm{Rx}}$ to $N_{\mathrm{MIMO}}=N_{\mathrm{Tx}} \cdot N_{\mathrm{Rx}}$, where the virtual phase center locations can be computed from a 

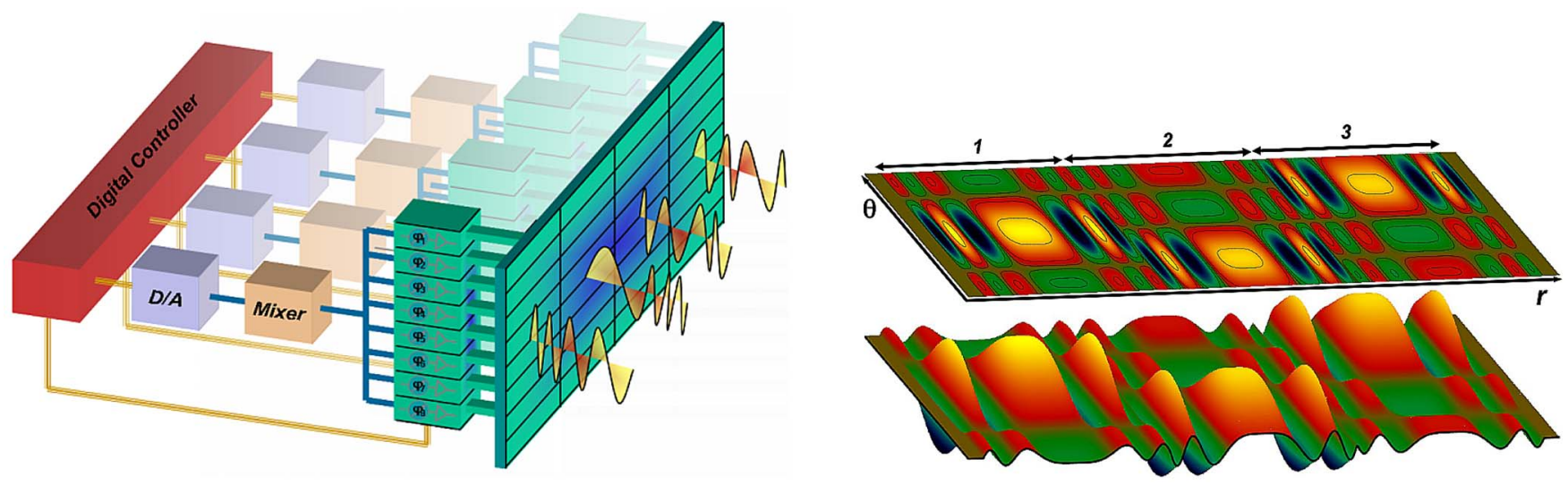

Fig. 1. MIMO-SAR architectures employ, in addition to multiple receivers, multiple transmit channels (left) to generate waveforms that are nonseparable with regard to their space-time coordinates (right). Conventional SAR systems are, in contrast, characterized by transmitting waveforms (or phase fronts) that can be written as the product of a time-only modulation function and a space-only angular antenna pattern (images from [9]).
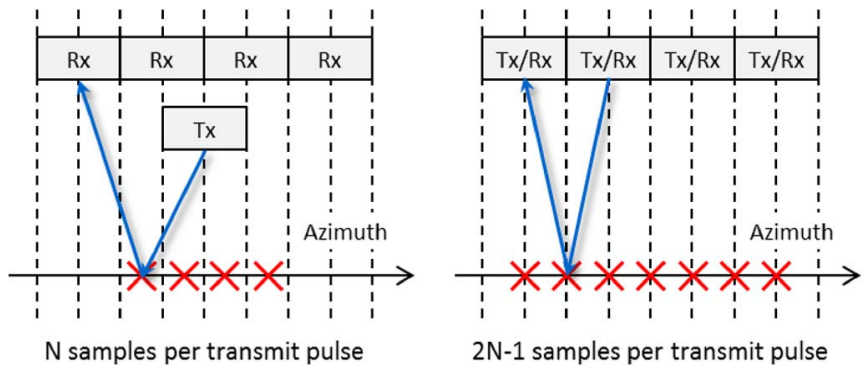

Fig. 2. Additional phase centers provided by a MIMO-SAR in the context of high-resolution wide-swath SAR imaging, ATI, and GMTI. (Left) azimuth phase centers for the case where multiple receive apertures are used in combination with a single transmitter (cf., [10]). (Right) MIMO-SAR where each antenna has, in addition, transmit capabilities (image adapted from [8]).

spatial convolution of the physical transmit and receive antenna positions [3]. Inspired by this diversity gain, [4] investigated the advantages of such a MIMO architecture to mitigate scintillations in the radar cross section (RCS) of point-like targets, and the edited book collection [5] compiled further analyses of MIMO concepts and processing techniques in the context of nonimaging radar systems. In the domain of real aperture radar imaging, additional transmit antennas were suggested to improve the angular resolution and to add interferometric or even tomographic capabilities in the context of a forward-looking imaging radar experiment [6]. A similar approach was later elaborated in more detail to improve the crosstrack resolution of a downward-looking 3-D imaging radar system [7].

In the field of SAR imaging, first suggestions of using a MIMO-SAR architecture with multiple transmit and multiple receive channels emerged in May 2006 in [8] and [9] (cf., Fig. 1). These early publications showed that the possible benefits of using multiple transmit channels together with a multichannel receiver range from an increased spatial coverage and geometric resolution to the improved suppression of range and azimuth ambiguities to the provision of additional baselines for ground moving target indication (GMTI) and interferometric applications. Fig. 2 illustrates, as an example, the additional azimuth phase centers that may arise from the transition of a multichannel receiver system (cf., [10]) to a
MIMO-SAR configuration. Here, the special case of a linear uniform array has been assumed, where the combined Tx/Rx elements are mutually displaced in the along-track direction. The extra phase centers provided by a MIMO-SAR yield additional and longer baselines for GMTI and along-track interferometry (ATI) applications and enable in the case of SAR imaging a reduction of the pulse repetition frequency (PRF) by a factor of two if compared to a single-channel transmitter. Hence, a wider image swath can be imaged as discussed in [2]. An elegant method to illustrate the virtual phase centers for an arbitrary arrangement of multiple spatially separated transmit and receive antennas has been presented in [11].

In [11] and [12], the possible application range of MIMOSAR was further extended to achieve superresolution in range by combining multiple image surveys of the same area acquired from slightly different incident angles [13]. For this, it was suggested to employ multiple transmit and receive platforms that are mutually separated by suitable cross-track displacements. The scene is illuminated by a set of transmitters radiating orthogonal waveforms that occupy the same range frequency band. The scattered echoes are then simultaneously recorded by an array of appropriately spaced receiver platforms. By this, a distributed MIMO-SAR system is configured. The proposed technique allows one to achieve a maximum theoretical range resolution improvement factor that is significantly greater than the number of operating SAR sensors by jointly exploiting both mono- and bistatic acquisitions [12].

Another interesting opportunity for a MIMO-SAR is fully polarimetric SAR imaging. All conventional fully polarimetric SAR systems transmit two orthogonal polarizations in subsequent pulse repetition intervals (PRIs), as illustrated on the lefthand side of Fig. 3. This implies an increase of the overall PRF, which causes, in turn, notable timing and/or range and/or azimuth ambiguity problems in the design of spaceborne SAR systems [14]. To avoid a deteriorated image quality in the delivered products, it has therefore become common practice to reduce both the swath width and the azimuth resolution as soon as a spaceborne SAR system is operated in fully polarimetric mode. As a possible way out of this dilemma, it was suggested in [15], and later again in [16], to transmit two orthogonally 


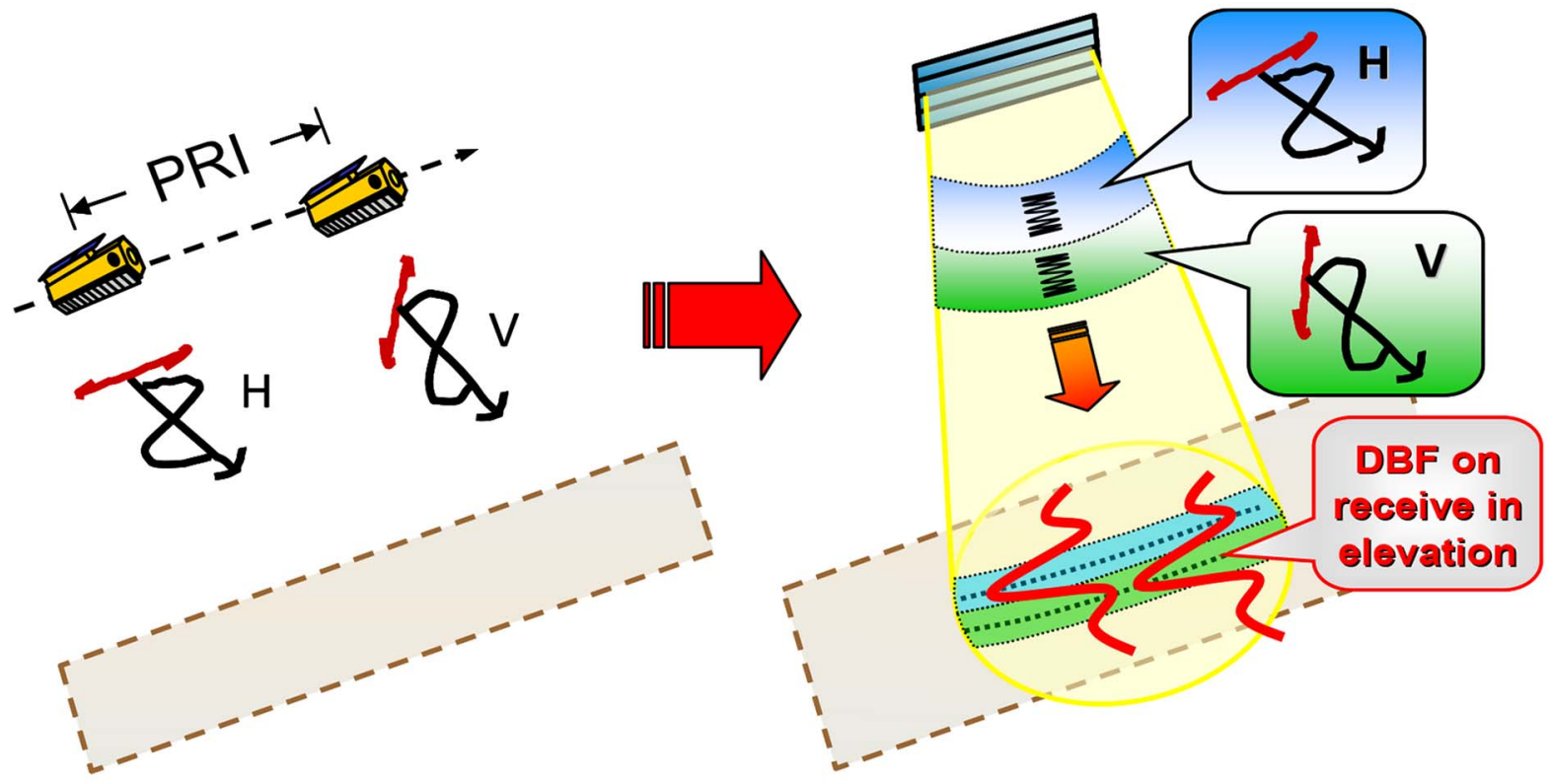

Fig. 3. MIMO-SAR for fully polarimetric wide-swath imaging (from [15]). (Left) Conventional SAR where two orthogonal polarizations (here, H and V) are transmitted in subsequent PRIs. The signals from each polarization must be sampled with a sufficiently high PRF to avoid azimuth ambiguities. As a result, the swath has typically to be narrowed to avoid both timing problems and range ambiguities. (Right) Two polarizations are transmitted within a single pulse (here, two subpulses are used, but other waveforms are also possible). The range ambiguous echoes from the polarized subpulses are then separated by DBF on receive (cf., Section IV). This polarimetric waveform encoding enables data acquisitions with multiple transmit polarizations during each PRI interval, thereby avoiding the necessity to increase the PRF for fully polarimetric operation. As argued in [15], this paves the way for a compact fully polarimetric high-resolution wide-coverage SAR imaging system.

polarized waves with appropriately chosen time modulations within each radar pulse. This avoids the otherwise unavoidable doubling of the PRF, and hence, it becomes possible to map a wider image swath without performance degradation. A possible embodiment of such a system is illustrated on the right-hand side of Fig. 3. We will see later that the performance improvement is not achieved without costs, and a higher antenna with DBF capabilities will be required to avoid mutual interferences between the received radar echoes from the two transmit signals.

\section{Orthogonal WaVeforms: The Great Confusion}

To fully exploit the MIMO-SAR opportunities outlined in the previous section, it is necessary to separate within the receiver the scattered radar echoes from the multiple transmit signals. To this end, several publications suggested to transmit mutually orthogonal waveforms. Notable confusion arose, however, what exactly is meant by "orthogonal."

\section{A. Orthogonality Without Shifts}

Some publications (cf., e.g., [17]-[20]) just require

$$
\int s_{i}^{*}(t) \cdot s_{j}(t) \cdot d t=0 \quad \text { if } \quad i \neq j
$$

where $s_{i}(t)$ and $s_{j}(t)$ are the transmitted signals from any two different apertures/channels. It had already been argued in [2] that such a condition allows perfect separation of the scattered waveforms from a single point target, but it is not sufficient to ensure reliable signal separation in case of spatially extended scattering scenarios. The reason for the poor performance is that the orthogonality is not ensured for arbitrary shifts between the different transmit signals. As a result, the energy from a distributed scatterer ensemble illuminated by a set of orthogonal waveform(s) does not vanish after range focusing but appears either smeared or at different positions [2]. This reasoning is immediately evident if one considers range focusing in the Fourier frequency domain as a bandpass filter with constant amplitude where the phase function is "matched" to one of the transmitted waveforms. As long as all transmitted orthogonal signals share the same frequency band, a mismatch of the phase will not affect the magnitude of the spectrum. According to Parseval's theorem, the output signals of all focusing filters will hence have the same power/energy regardless of whether a matched or an unmatched signal is present. This means that the signal energy from all unmatched waveforms is still present in the focused signal. Depending on the number of transmitters, the smeared energy from the orthogonal waveforms may therefore even exceed the energy of the focused image response. The interested reader may consult Appendix A for a more rigorous analytic derivation of this fundamental result.

Despite this apparent limitation, it has unfortunately to be noted that several recent peer-reviewed journal publications neglected (or underestimated) the conservation of the signal energy from the orthogonal waveforms [17], [18], [20]. It seems therefore necessary to illustrate the deteriorating effects that may arise from the mere usage of orthogonal waveforms satisfying (1). For this, four simulations have been performed.

Fig. 4 shows the effect of using a slightly adapted pair of upand down-chirps as orthogonal transmit waveforms. To satisfy the orthogonality condition in (1), the second chirp has been marginally modified by adding a small additional signal as 


\section{Original Scene}

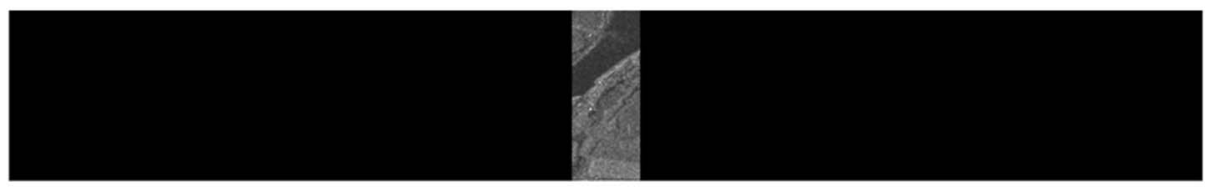

Matched Filter Result (only matched channel present)

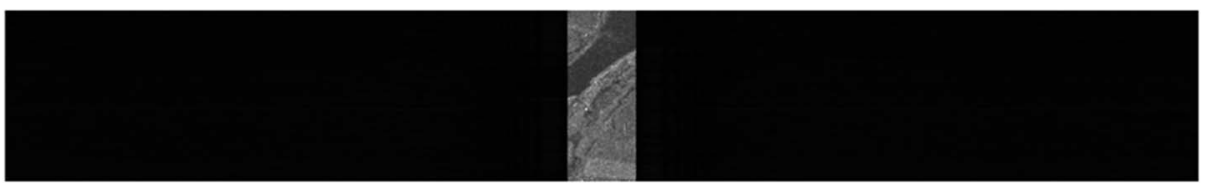

Matched Filter Result (also orthogonal channel present)

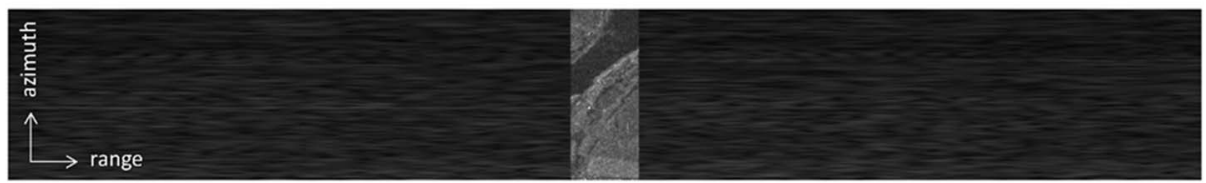

Power Profiles (average across azimuth)

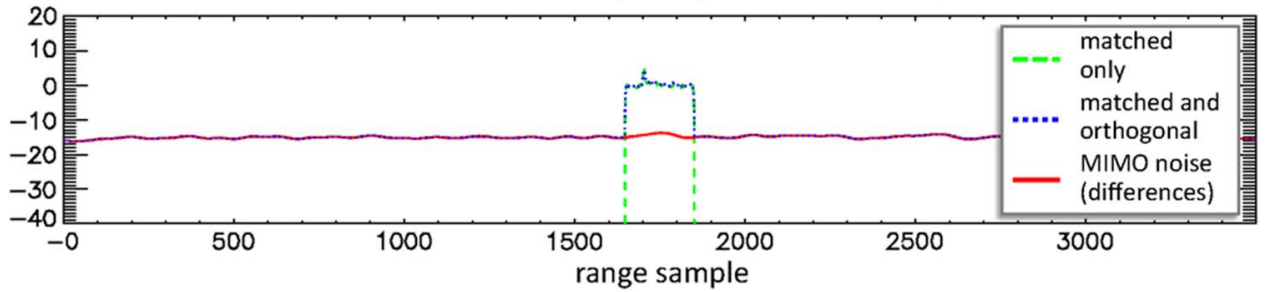

Fig. 4. Simulation of MIMO-SAR imaging based on the simultaneous transmission of up- and down-chirps. The scene has a short range extension (201 pixels) if compared to the length of the range chirp (7680 samples). (Top) Original scene. (Upper middle) Matched filter result in case that only the up-chirp is present. (Lower middle) Matched filter result in case that both the up- and down-chirps are present. (Bottom) Dashed green and dotted blue style curves show the range profiles of the azimuth averaged power of the second and third images, respectively, while the solid red profile shows the azimuth averaged power of the difference between the two images.

explained in Appendix B. ${ }^{1}$ In this first simulation, a very short scene extension of only 201 pixels has been emulated by setting the remaining pixel values of the selected input image to zero. The top image of Fig. 4 shows a picture of the original scene that provided the input for the simulation. The second image of Fig. 4 shows the matched filter result if only one waveform (up-chirp) with a pulse length of 7680 samples is transmitted. It can be seen that the scene is well reconstructed within the chirp bandwidth. The third image of Fig. 4 shows the matched filter result (for the up-chirp) if both the up- and down-chirps are transmitted at the same time. Since the scene extension is much shorter than the length of the transmitted chirp signal, only a slight image degradation can be seen. This is due to the fact that the leaked signal energy from the orthogonal waveform (down-chirp in this case) is distributed among the whole chirp length, and as a consequence of the short scene extension, only a small fraction of this leaked energy overlaps with the scene. The energy leakage from the orthogonal signal can be seen in the third image of Fig. 4 by a slight degradation of the black values, especially outside the original scene extension. For a quantitative analysis, three power profiles have been added at

\footnotetext{
${ }^{1}$ The results in Figs. 4 and 5 are very similar whether or not the small correction signal is present in the second transmitted waveform. This has been verified by performing the simulations once with and once without the correction signal, which resulted in almost indistinguishable results. We therefore speak in the following only of up- and down-chirps, keeping in mind that the second chirp has been slightly modified to satisfy (1).
}

the bottom of Fig. 4. The dashed green and dotted blue profiles show the azimuth averaged power of the second (only matched channel) and third (matched + orthogonal channel) images, respectively, while the red profile shows the azimuth averaged power of the difference between the two images. The red curve can therefore be regarded as representing the additional noise that is caused by the presence of the second transmit channel. This noise level is approximately $15 \mathrm{~dB}$ below the signal level, which fits well its expected value that is provided by the ratio between the scene extension (201 samples) and the pulse length (7680 samples).

A completely different picture arises, however, if the scene width exceeds the length of the chirped signal. This is the typical case for most spaceborne SAR imaging systems like ERS-1/2, Sentinel-1, ALOS, Radarsat-2, TanDEM-X, etc. ${ }^{2}$ For this, Fig. 5 shows the same simulations as before but now for a scene that is much wider than the chirp length. While the scene is well reconstructed if only the matched signal is transmitted (middle image of Fig. 5), a significant degradation of the image quality and contrast can be noted for the case where the orthogonal down-chirp is transmitted together with the up-chirp (lower image in Fig. 5). From this, it should be clear that the mere use of orthogonal transmit signals is, in

\footnotetext{
${ }^{2}$ An exception is the high-resolution spotlight mode of COSMO-Skymed which employs a deramping technique to decrease the data rate and volume of the large bandwidth received signal.
} 


\section{Original Scene}

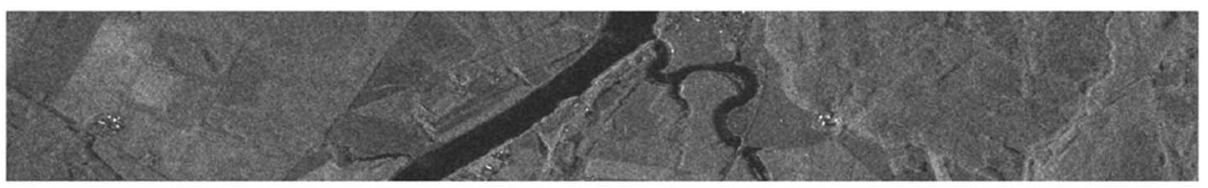

Matched Filter Result (only matched channel present)

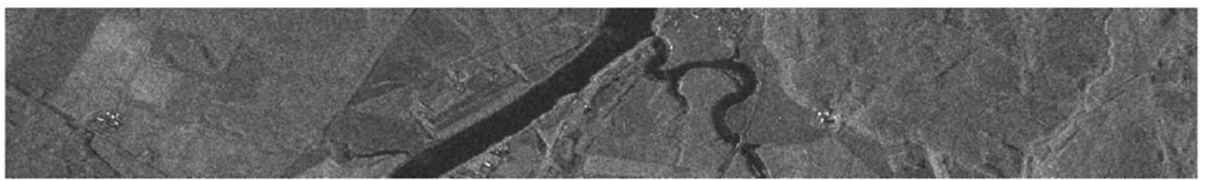

Matched Filter Result (also orthogonal channel present)

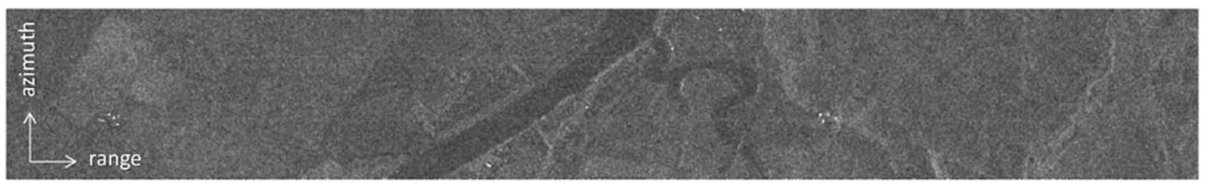

Power Profiles (average across azimuth)

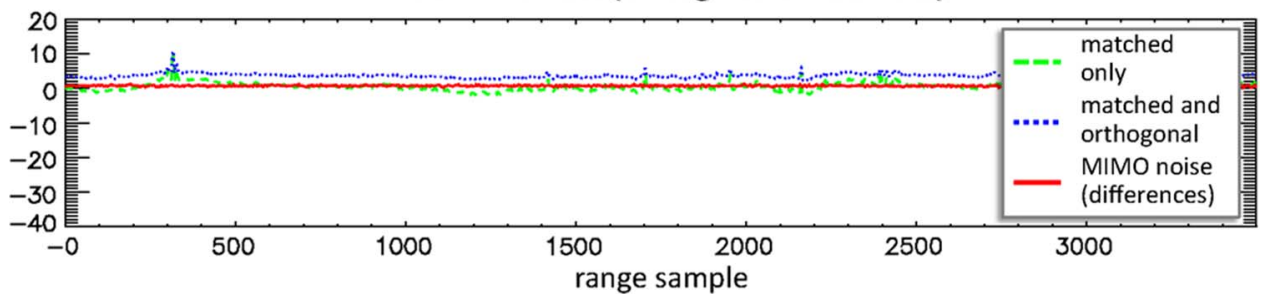

Fig. 5. Same as in Fig. 4 but for a simulated scene extension in range which significantly exceeds the chirp length. The increased scene width leads to a significant rise of the MIMO noise which has become as large as the signal level.

general, not sufficient for the successful implementation of a high-quality wide-swath MIMO-SAR system.

Orthogonal waveforms like the investigated up- and downchirps (or more sophisticated waveforms) may, however, be of interest for special scenarios. One example is a bistatic ground-based MIMO-ISAR system, where the imaged scene size is typically limited by the rapid decay of the backscattered signal with range. By choosing transmit pulses of sufficient length, a high-quality imaging with low energy leakage from the orthogonal channels can be achieved [19]. Another possible example is the imaging of a sparse set of strong scatterers embedded in a large scene of low backscatter. Such a scenario may, for example, apply to a calm sea with some ships floating on the water. In this case, the energy leakage from the orthogonal waveforms is distributed over a wide area, and the typically strong radar echoes from the ships remain well above the slightly increased noise level. An appropriately designed MIMO-SAR system may, in this case, provide an improved detection and parameter estimation performance.

Several recent publications argued that up- and down-chirps are not suitable for the implementation of a MIMO-SAR system. As an alternative, more sophisticated waveforms were suggested in [20] and [22]. The proposed waveforms consist essentially of the concatenation of multiple subpulses, where each subpulse is again composed of the linear superposition of multiple chirped signals, each covering a small fraction of the overall bandwidth. Fig. 6 illustrates a pair of these waveforms in a time-frequency diagram (cf., [20] for more details).
Fig. 7 shows the same set of simulations as in Fig. 4, but now, the orthogonal waveforms from Fig. 6 have been employed instead of up- and down-chirps. The scene extension and the total pulse length are again 201 and 7680 samples, respectively. The image quality for the narrow scene is again acceptable even in the presence of the orthogonal signal, but one can note as before a slight degradation of the dynamic range. Note that the black areas outside the original scene extension are now filled by a periodic pattern. This pattern is a consequence of the suggested OFDM waveforms that are composed of multiple chirped subpulses with partially overlapping spectra. A close inspection of the middle image reveals that a similar pattern is even visible here but at a lower level. This image degradation becomes also evident from the azimuth averaged power profiles shown at the bottom of Fig. 7.

A completely different picture arises if the scene size exceeds the pulse length. The corresponding simulation results are shown in Fig. 8. It can be seen that the matched filter output now suffers from a severe performance degradation even for the case where no orthogonal signal is present. This can be explained again by the repeated subpulse structure which covers overlapping frequency bands. It should therefore be clear that, as it was the case for the mere use of up- and down-chirps, only very narrow scenes can be mapped by the suggested OFDM waveforms without suffering a severe performance degradation. To conclude, the proposed OFDM waveforms are unfortunately not suitable for the implementation of a spaceborne wide-swath SAR imaging system. The same applies, in general, to all other 

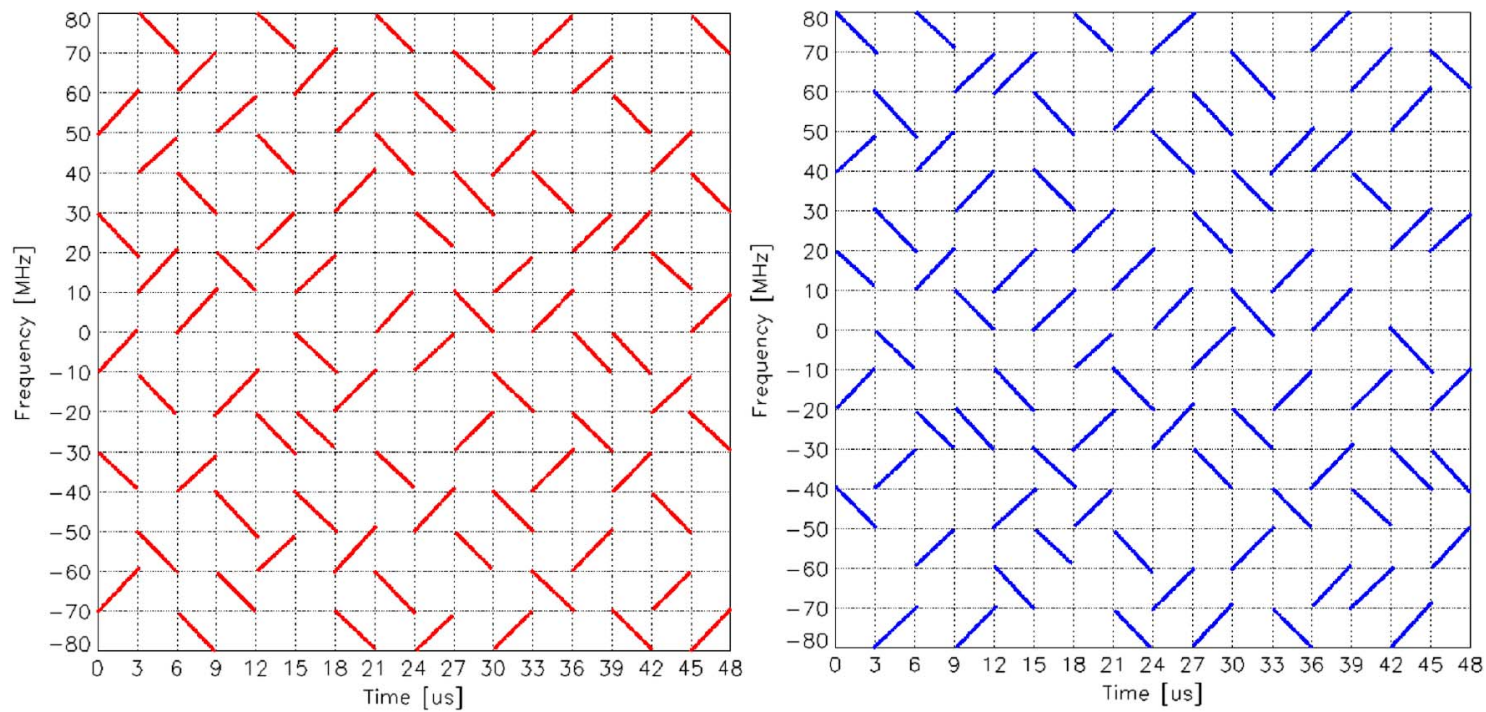

Fig. 6. OFDM waveforms as suggested in [20].

Original Scene

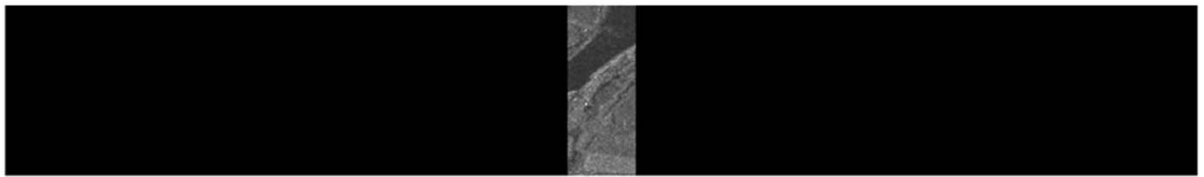

Matched Filter Result (only matched channel present)

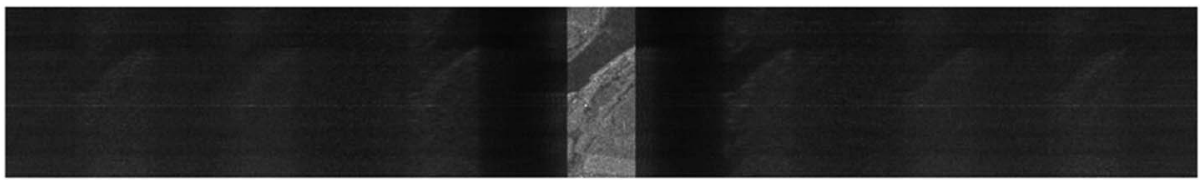

Matched Filter Result (also orthogonal channel present)

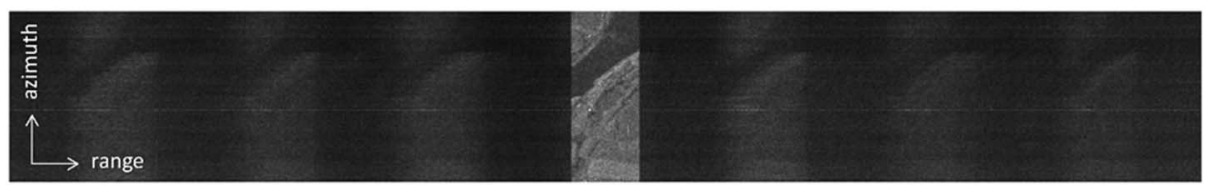

Power Profiles (average across azimuth)

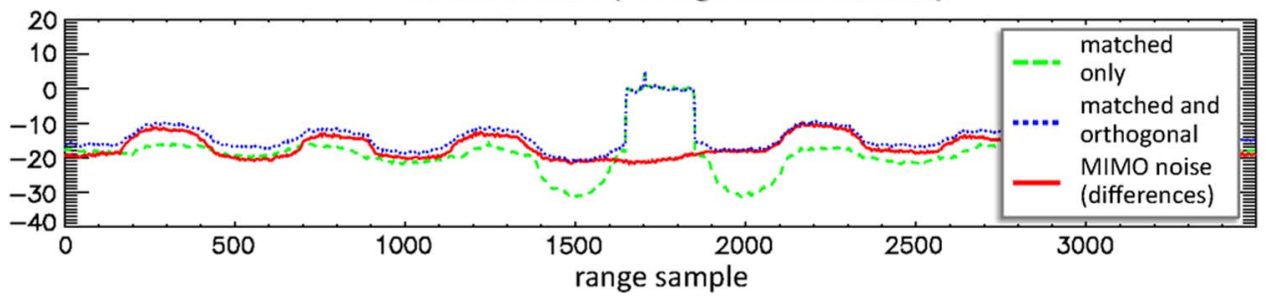

Fig. 7. Simulation of MIMO-SAR imaging based on the simultaneous transmission of the waveforms shown in Fig. 6. The scene has again a short range extension (201 samples) if compared to the length of the transmitted OFDM waveforms (7680 samples). (Top) Original scene. (Upper middle) Matched filter result for transmitting only the left OFDM waveform from Fig. 6. (Lower middle) Matched filter result in case that both OFDM waveforms were transmitted. (Bottom) Range profiles of the azimuth averaged power for the second image (dashed green), the third image (dotted blue), and the difference between the second and third images (solid red).

suggested MIMO-SAR waveforms that are based on a mere use of (1) for their design.

\section{B. Orthogonality for Arbitrary Shifts}

To avoid the inherent limitations and problems pointed out in the previous section, some other publications (cf., e.g., [11],
[21], and [23]) require the orthogonality for arbitrary time shifts $\tau$ between the signals $s_{i}(t)$ and $s_{j}(t)$

$$
\int s_{i}^{*}(t) \cdot s_{j}(t+\tau) \cdot d t=0 \quad \forall \quad \tau \in \mathbb{R}, i \neq j .
$$

This enables a perfect signal separation also in case of a distributed scatterer scenario (possible effects from Doppler 


\section{Original Scene}

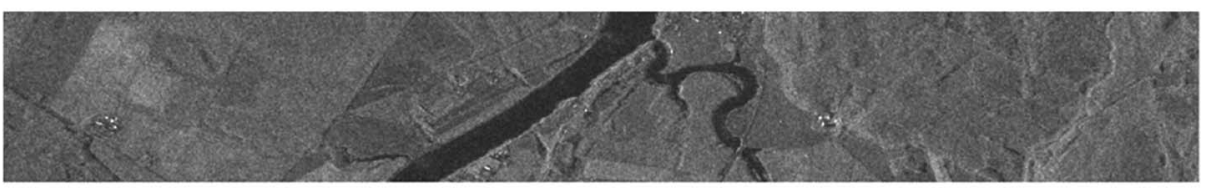

Matched Filter Result (only matched channel present)

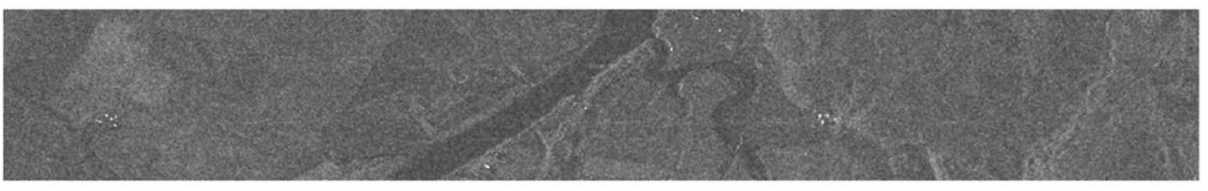

Matched Filter Result (also orthogonal channel present)

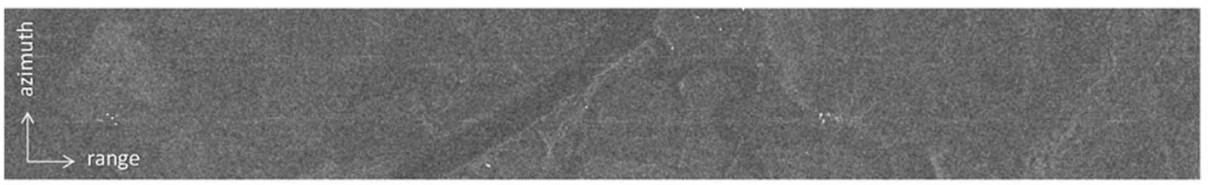

Power Profiles (average across azimuth)

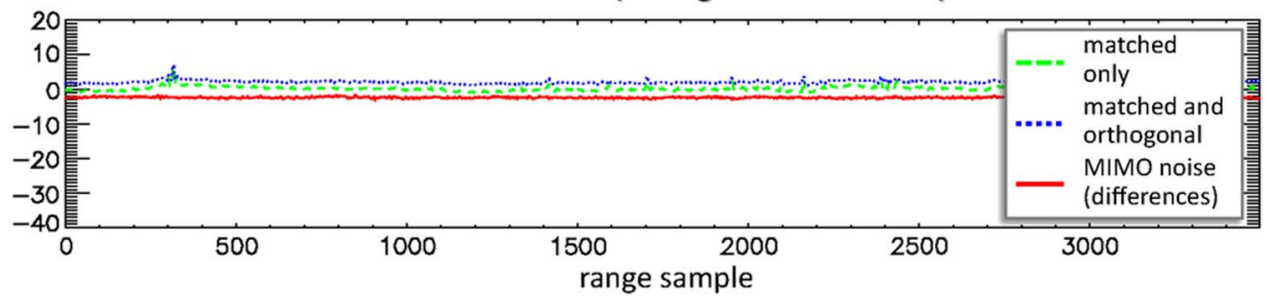

Fig. 8. Same as in Fig. 7 but for a scene extension in range which exceeds the length of the transmitted waveform.

shifts will be discussed later). However, an immediate consequence of this requirement is that $s_{i}(t)$ and $s_{j}(t)$ must have nonoverlapping spectral support. This is evident from the crosscorrelation theorem

$$
s_{i}(t) \otimes s_{j}(t)=\mathcal{F}^{-1}\left[S_{i}^{*}(f) \cdot S_{j}(f)\right]
$$

where $\otimes$ is a short hand for the cross-correlation integral of (2), $\mathcal{F}^{-1}$ denotes the inverse Fourier transform, and $S_{i}(f)$ and $S_{j}(f)$ are the Fourier transforms of $s_{i}(t)$ and $s_{j}(t)$, respectively. The cross-correlation theorem is comparable to the wellknown convolution theorem and states that the cross-correlation of two functions can be expressed (up to a sign change in the conjugate complex function) in the frequency domain as the product of their individual Fourier transforms.

It is evident from (3) that (2) can only be fulfilled if the product between $S_{i}(f)$ and $S_{j}(f)$ vanishes for all $f$. This implies that $S_{i}(f)$ and $S_{j}(f)$ have no common spectral support. The inevitable restriction to mutually distinct frequency bands therefore excludes shift-orthogonal waveforms from the use in scenarios where full spectral overlap between the radar echoes from multiple transmit signals is required. A coherent combination of spectrally overlapping radar echoes is, however, the basis of all MIMO-SAR applications mentioned in Section II. The mere use of shift-orthogonal waveforms according to (2) is therefore again not a viable solution for the implementation of future MIMO-SAR systems.

\section{Solution: Short-Term Shift-OrThogonal WAVEFORMS IN COMBINATION WiTH DBF ON RECEIVE}

To overcome the fundamental challenges described in the previous section, it was suggested in [2], [8], and [9] to employ especially designed waveforms together with DBF on receive in the elevation direction. The basic idea underlying this proposal can be expressed in a more general form by a restricted shiftorthogonality condition

$$
\int h(\tau) \cdot s_{i}^{*}(t) \cdot s_{j}(t+\tau) \cdot d t=0 \quad \forall \tau \in \mathbb{R}, i \neq j
$$

where, in comparison to (2), an additional weighting function $h(\tau)$ has been included which depends on the relative time shift $\tau{ }^{3}$ The novel function $h(\tau)$ is, as it will soon become clear, closely linked to the side-looking data acquisition geometry of a ground imaging radar.

To understand the significance of $h(\tau)$, note first that there exists in a side-looking imaging radar for each scatterer on the ground a direct correspondence between the angle of arrival of the scattered wave and the associated radar signal delay. This means that signals from targets at different ranges arrive also from different look angles. As a result, the echoes from targets with sufficient mutual range delay $\tau$ can be separated in a multichannel receiver by an appropriate beamforming in the elevation direction. This situation is illustrated in Fig. 9,

\footnotetext{
${ }^{3} \mathrm{~A}$ possible dependence of $h(\tau)$ on $t$ is neglected here to ease understanding.
} 


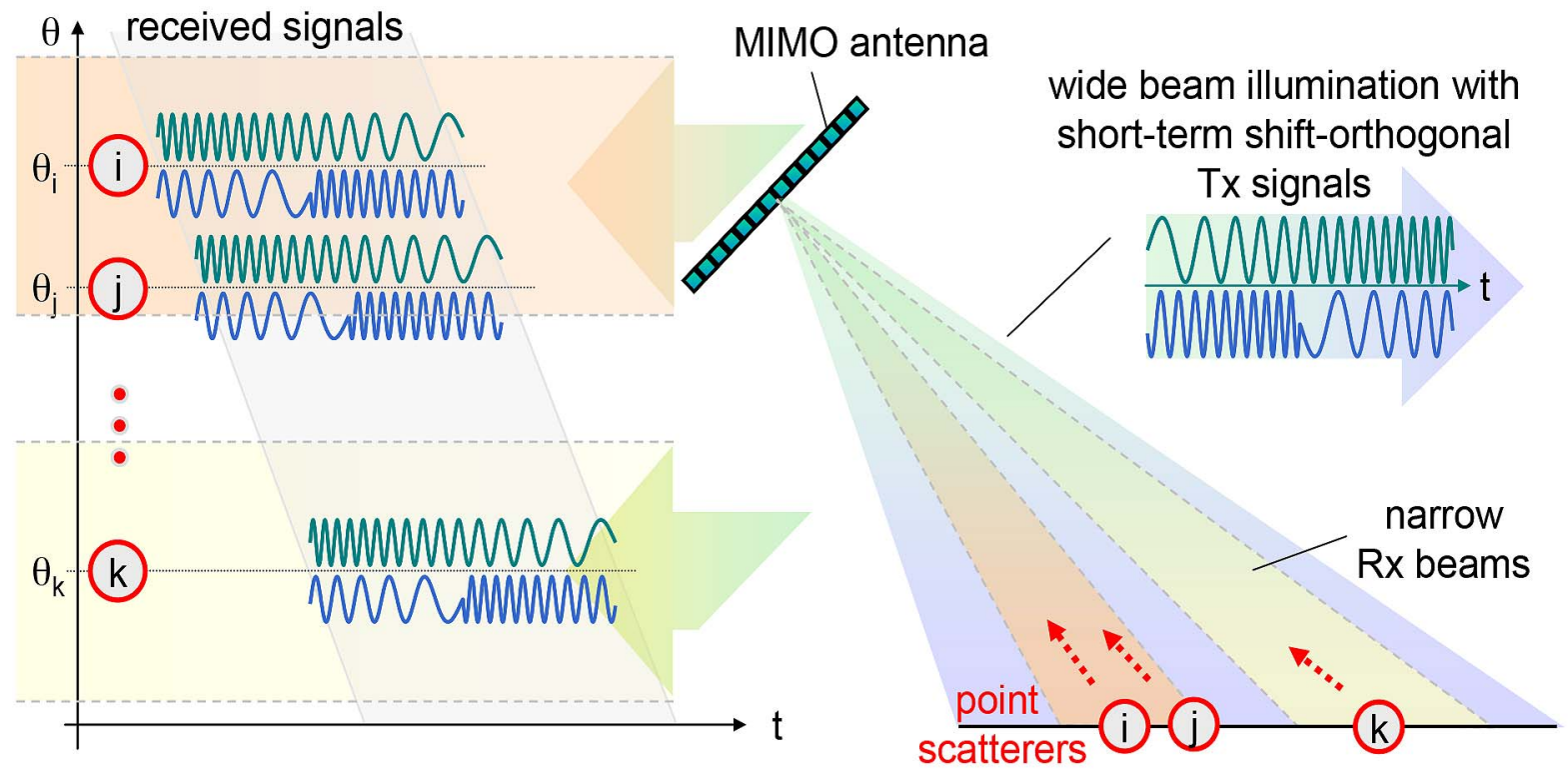

Fig. 9. MIMO-SAR based on two short-term shift-orthogonal transmit signals. The waveforms are designed such that their cross-correlation vanishes for small time offsets (as it applies to scatterers $i$ and $j$ ). For larger time offsets, the otherwise correlated signals are separated via narrow Rx beams (as it applies to scatterers $i$ and $k$ ). This exploitation of the side-looking imaging geometry together with appropriately designed transmit waveforms enables the simultaneous use of multiple Tx signals and the unambiguous separation of their radar echoes also in the realistic case of a distributed scatterer scenario.

which shows on the lower right a set of point scatterers that are illuminated by two orthogonal Tx waveforms. This pair of transmit signals has been chosen in accordance to (4) for a specific weighting function $h(\tau)$ to be defined later. The radar echoes from the individual point scatterers arrive then according to the different pulse travel times at different instances of time as shown in the upper left of Fig. 9, where the pulse echoes from the neighboring near range scatterers $i$ and $j$ arrive significantly before those from the far range scatterer $k$. DBF on receive enables a reliable separation (or spatial filtering) of the mutually overlapping radar echoes $\{i, j\}$ from those emanating from the point scatterer $k$. This is illustrated in Fig. 9 by the distinct yellow and orange beams. To ensure a reliable signal separation, a sufficient number of receiver channels should be available, and the antenna height of an optimally oriented antenna $h_{\text {ant }}$ should exceed [2]

$$
h_{\mathrm{ant}} \geq \frac{2 \lambda \cdot r_{\mathrm{far}} \cdot \tan \left(\theta_{\mathrm{inc}, \max }\right)}{c \cdot \Delta \tau}
$$

where $\lambda$ is the wavelength, $r_{\mathrm{far}}$ is the far range distance, $\theta_{\text {inc,max }}$ is the maximum incident angle, $c$ is the velocity of light, and $\Delta \tau$ is the minimum time interval between the arriving signals that should be separated by DBF on receive (cf., Fig. 10). For small antenna heights, an accurate null steering may be used to improve the spatial filtering [2], [24], [25].

On the other hand, the echoes from the scatterers $i$ and $j$ cannot be mutually separated by the spatial beamforming since they are located too close together. The only means to avoid a mutual interference of the radar echoes for these closely spaced scatterers is an appropriate choice of the transmitted waveforms such that they are mutually orthogonal within the time interval that cannot be resolved by the spatial beamforming in elevation. For this, we consider in (4) the function

$$
h(\tau)=\operatorname{rect}\left(\frac{\tau}{\Delta \tau}\right)
$$

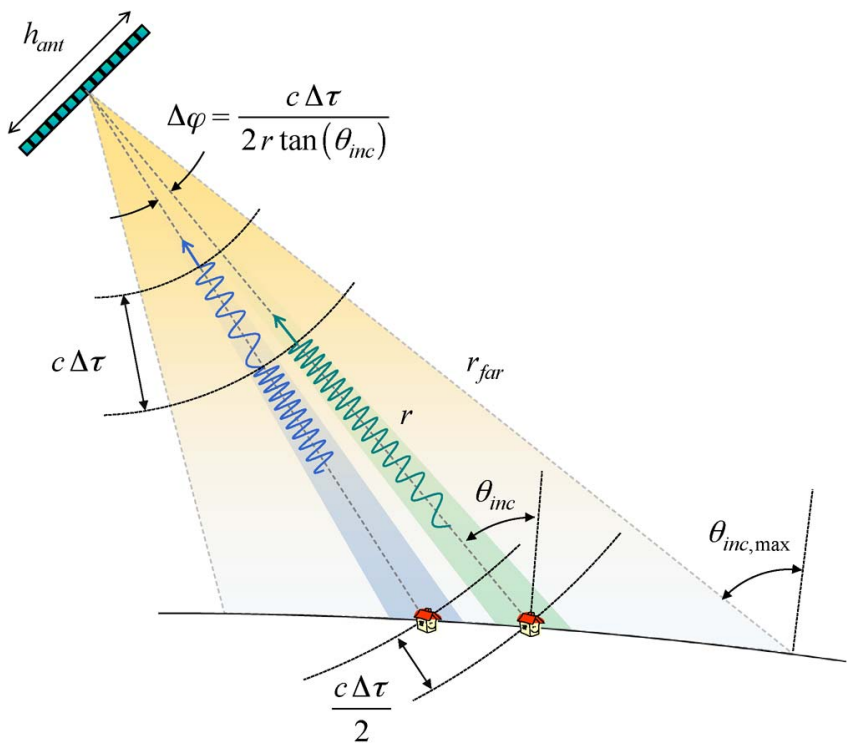

Fig. 10. Illustration of the derivation of the minimum antenna height in (5). A wide scene is illuminated by two orthogonal waveforms. The waveforms have been chosen such that they are orthogonal for mutual time shifts that are shorter than $\Delta \tau$. Hence, no interference occurs for scatterers whose slant range difference is smaller than $c \Delta \tau / 2$. To avoid mutual interference for scatterers that are separated in slant range by $c \Delta \tau / 2$ and more, a sufficiently narrow antenna beam is needed, which substitutes the temporal orthogonality by a spatial filtering. To derive the maximum allowed beamwidth, one needs, in turn, the angular separation $\Delta \varphi$ from which the radar echoes arrive for targets separated in slant range by $c \Delta \tau / 2$. This angular separation $\Delta \varphi$ is easily derived from the shown geometry by using straightforward linear approximations and depends besides $\Delta \tau$ on the incident angle $\theta_{\text {inc }}$ and the slant range $r$ as displayed in the formula on the top. The narrowest angle $\Delta \varphi_{\text {min }}$ results for scatterers at the far range border of the illuminated swath. From this, the minimum antenna height in (5) is immediately derived by using the standard antenna beamwidth approximation $\Delta \varphi_{\min } \approx \lambda / h_{\text {ant }}$.

which is one within the interval $[-\Delta \tau / 2, \Delta \tau / 2]$ and zero elsewhere. This means that the cross-correlation on the left-hand 
side of (2) has to vanish only for mutual time shifts $\tau$ that are smaller than $\Delta \tau / 2$ :

$$
\int s_{i}^{*}(t) \cdot s_{j}(t+\tau) \cdot d t=0 \quad \forall \tau \in\left[-\frac{\Delta \tau}{2}, \frac{\Delta \tau}{2}\right], i \neq j .
$$

This specific choice of $h(\tau)$ leads to a short-term shiftorthogonal waveform and offers a new degree of freedom to reintroduce spectral overlap between the orthogonal waveforms $S_{i}(f)$ and $S_{j}(f)$.

One specific choice of such a short-term shift-orthogonal waveform could be based on mutually time-shifted chirped pulses as already suggested in [2], [8], and [9]. In these references, it was proposed to use for each desired transmit channel a conventional chirped signal, each covering the whole range bandwidth. To avoid interferences between the individual transmit signals and to enable a reliable separation of their radar echoes by DBF on receive in elevation, it was further suggested to transmit the individual chirped pulses in subsequent order, i.e., there was no temporal overlap between the waveforms of the orthogonal transmit channels. The individual waveforms transmitted by the different channels were then denoted as subpulses. A drawback of this technique arises, however, if the subpulses are to be transmitted from different portions of a large antenna array where each of these subarrays is fed by a separate set of $T / R$ modules. Such a scenario is, for example, desired if additional phase centers are to be created for multibaseline interferometry, tomography, improved GMTI, and high-resolution wide-swath SAR imaging applications (cf., illustration in Fig. 2). Since only a portion of all available T/R modules is used for each subpulse, it is therefore necessary to increase the peak power from each T/R module to obtain the same average transmit power as a conventional SAR system which transmits with all T/R modules at the same time. It should be evident that this drawback becomes more severe with more transmit channels employed. ${ }^{4}$

To overcome this possible limitation, an alternative approach is illustrated in Fig. 11, which employs a signal with linear frequency modulation for $s_{i}(t)$ and a second signal $s_{j}(t)$ with a sufficient offset in its instantaneous frequencies. The advantage of this new type of waveform, if compared to the previously suggested use of subpulses, is that multiple waveforms are transmitted at exactly the same time. By this, the peak power requirements for the signal transmissions can be reduced. The required frequency offset can, in principle, be obtained by a mere cyclic shift of the chirped signal $s_{j}(t)$. This simple method also allows a straightforward extension to an arbitrary number of shift-orthogonal waveforms. The special choice for $s_{i}(t)$ and $s_{j}(t)$ ensures that there is no interference between the signal returns for closely separated scatterers, while scatterers with a larger mutual separation would cause an interference

\footnotetext{
${ }^{4}$ To avoid such an undue increase of the peak power requirements, it was suggested in [2] to transmit at each instant of time with all antenna elements but to switch from subpulse to subpulse between appropriately designed antenna beams in order to achieve the transmit diversity gain desired for high-resolution wide-swath MIMO-SAR imaging.
}

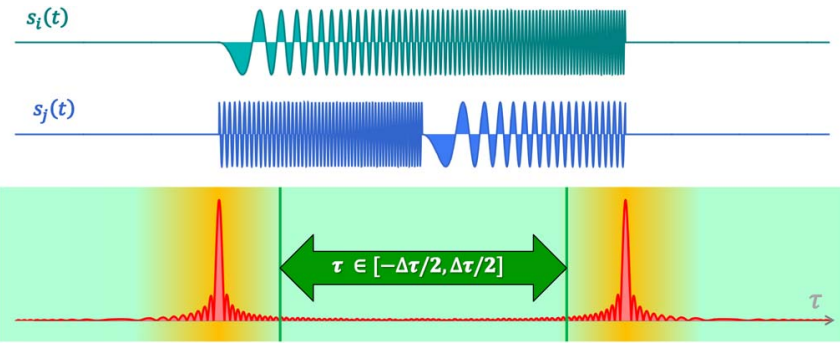

Fig. 11. Illustration of short-term shift orthogonality. (Top) Two waveforms $s_{i}(t)$ and $s_{j}(t)$ cover the same overall frequency band but are offset in their instantaneous frequencies. (Bottom) Cross-correlation shows that the two waveforms are (almost) orthogonal for mutual time shifts within the interval $\tau \in[-\Delta \tau / 2, \Delta \tau / 2]$. The signals $s_{i}(t)$ and $s_{j}(t)$ therefore satisfy the restricted shift-orthogonality condition from (4) for $h(\tau)=\operatorname{rect}(\tau / \Delta \tau)$. The remaining cross-correlation within the interval $\tau \in[-\Delta \tau / 2, \Delta \tau / 2]$ is typically much smaller as in this illustration due to the large time-bandwidth product employed for spaceborne SAR sensors. The acceptable level for the integrated leakage energy from the orthogonal channel(s) depends on the intended application, but it is expected that an easily obtainable suppression of $20 \mathrm{~dB}$ with respect to the integrated energy in the matched channel will be more than sufficient for most spaceborne MIMO-SAR imaging applications.

between the received signals. ${ }^{5}$ The interference for the longer shifts can, however, be avoided by the aforementioned spatial filtering, thereby exploiting the specific space-time structure of the received signal in a side-looking imaging radar. The spatial filtering is, as illustrated in Fig. 9, equivalent to a DBF on receive in elevation.

To summarize, the radar echoes from closely spaced scatterers are separated in the temporal domain by using short-term shift-orthogonal waveforms for the different transmit channels, while the echoes from widely spaced scatterers are separated in the spatial domain by DBF on receive. The combination of appropriately designed short-term shift-orthogonal Tx waveforms with $\mathrm{DBF}$ on receive in elevation hence enables the implementation of a MIMO-SAR not only for an academic scenario consisting of one or a few closely spaced scatterers as considered in several recent MIMO-SAR publications, but also for a realistic spaceborne SAR scenario where an extended scene is filled with a large number of distributed scatterers and where the swath width exceeds the spatial extension of the transmitted pulse.

\section{INFORMATION CUBE}

Further insights into the intricacies and challenges of MIMOSAR systems can be obtained by introducing the concept of the information cube which provides a concise visualization of the information collected by a multiaperture radar receiver. For this, we start from a conventional radar with a singlechannel receiver. The upper right side of Fig. 12 shows the corresponding information cube filled with a green surface. To understand the significance of this surface, let us first assume that the radar transmits a pulse with liner frequency modulation (here, down-chirp) and the scene consists of a single scatterer. In this case, a single chirped signal would be received, which approaches the antenna from a fixed angle of arrival. The

\footnotetext{
${ }^{5}$ This special choice of waveforms provides, moreover, maximum Doppler tolerance, avoiding, for example, the problem outlined in Section $\mathrm{V}$ where a portion of the signal energy from one channel may be leaked into the other channel if a broad Doppler spectrum has to be acquired.
} 


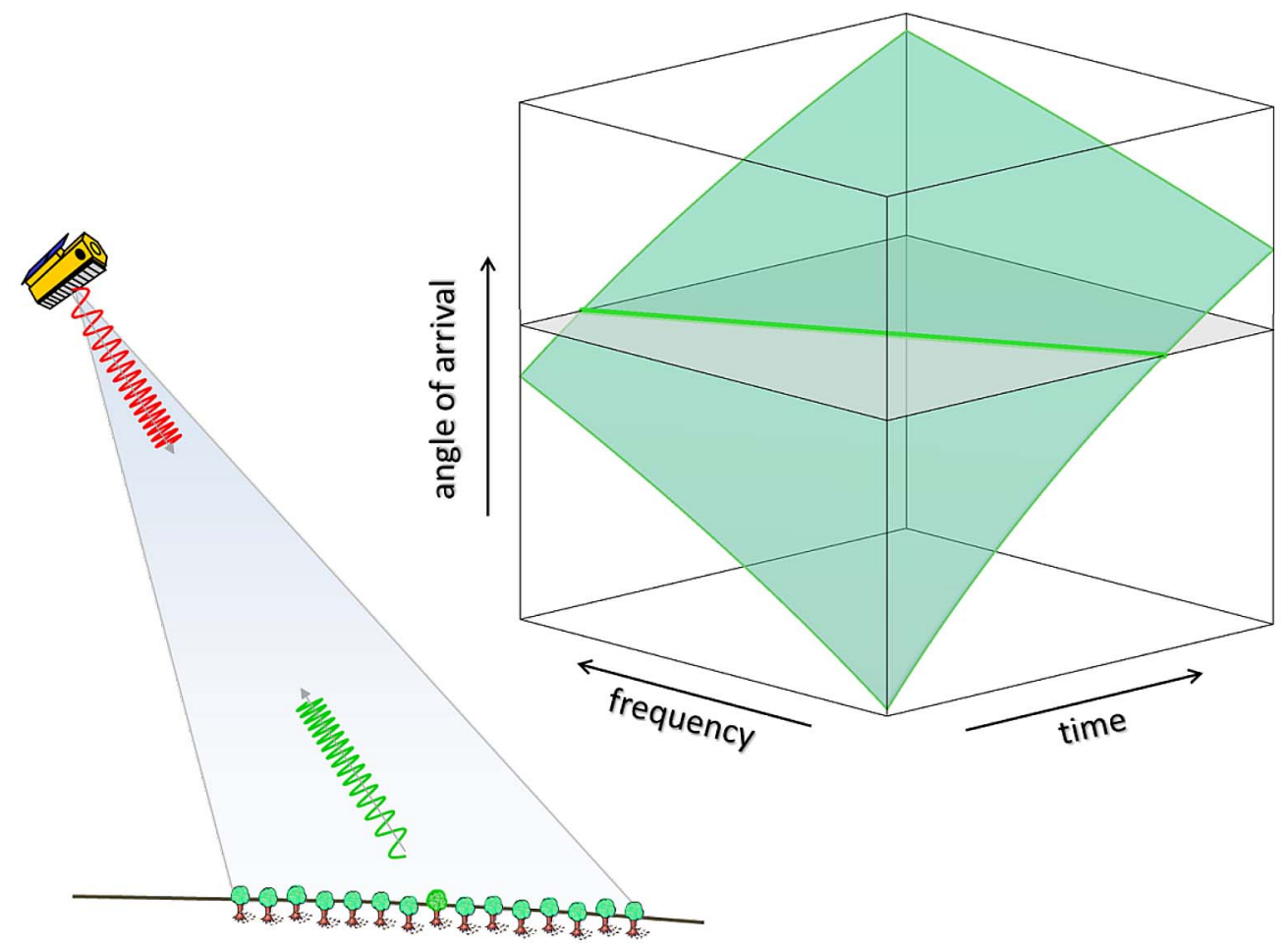

Fig. 12. Information cube for a conventional single-channel SAR. (Left) An extended scene is illuminated by a down-chirp, and the scattered echoes are recorded by a single-channel receiver. (Right) Signals received from a distributed scatterer scenario are represented in the information cube by a curved surface. For each constant angle of arrival (cf., horizontal plane), the received signal is a linear FM pulse (down-chirp in this case). Note that the depicted 3-D representation of the information cube has, for better illustration, been limited in both the covered time interval and the angles of arrival. The shown surface therefore represents only a fraction of the total swath signal.
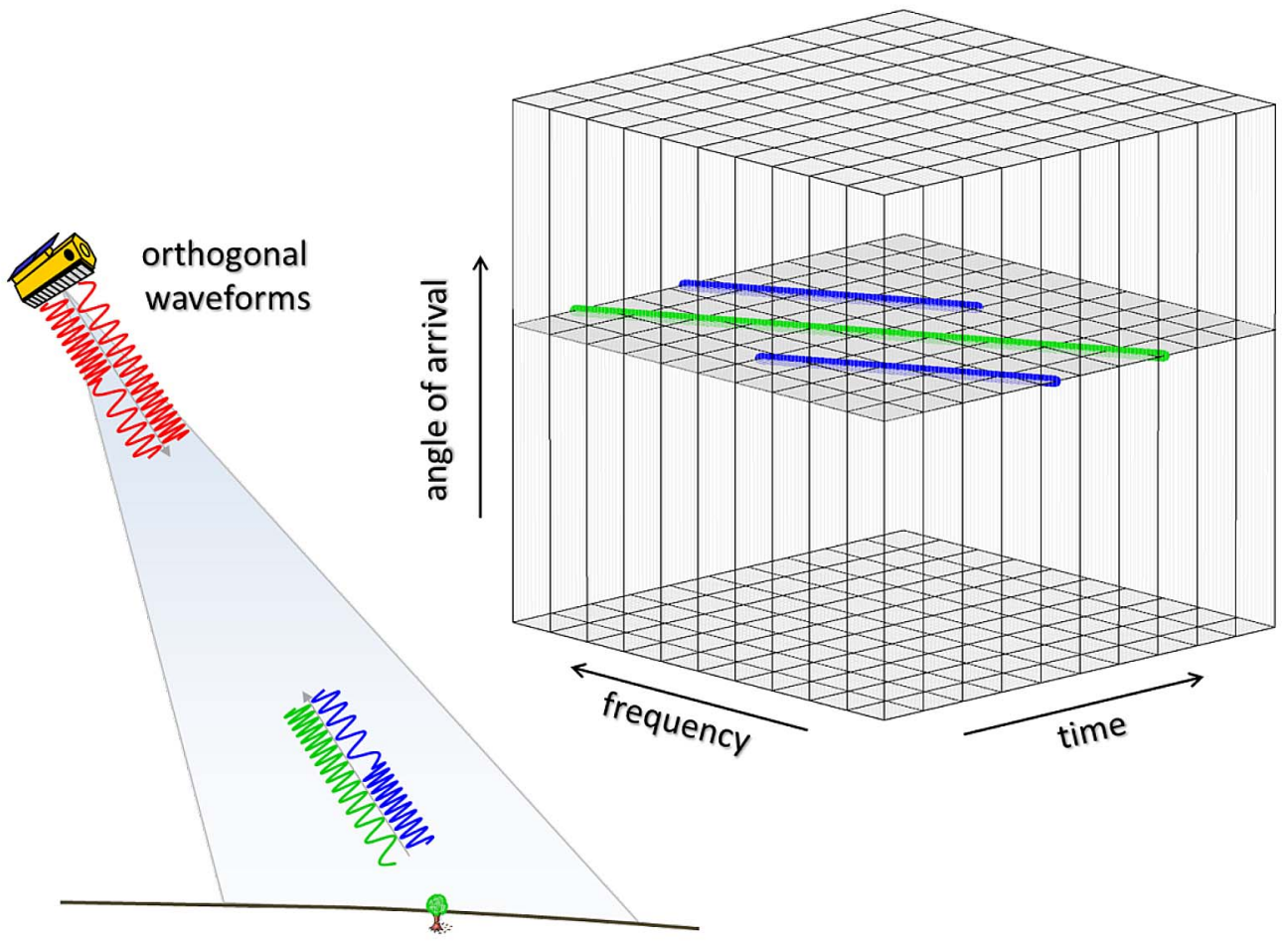

Fig. 13. Information cube in case of the simultaneous transmission and reception of two orthogonal waveforms received from a single point-like scatterer.

constant angle of arrival is indicated in the information cube of Fig. 12 by the gray horizontal plane. Within this plane, the chirped signal can be seen by a linear frequency decrease as the time proceeds (due to the transmitted down-chirp). Let us now assume that the scene contains additional scatterers at different ranges. In this case, additional chirped signals would be 


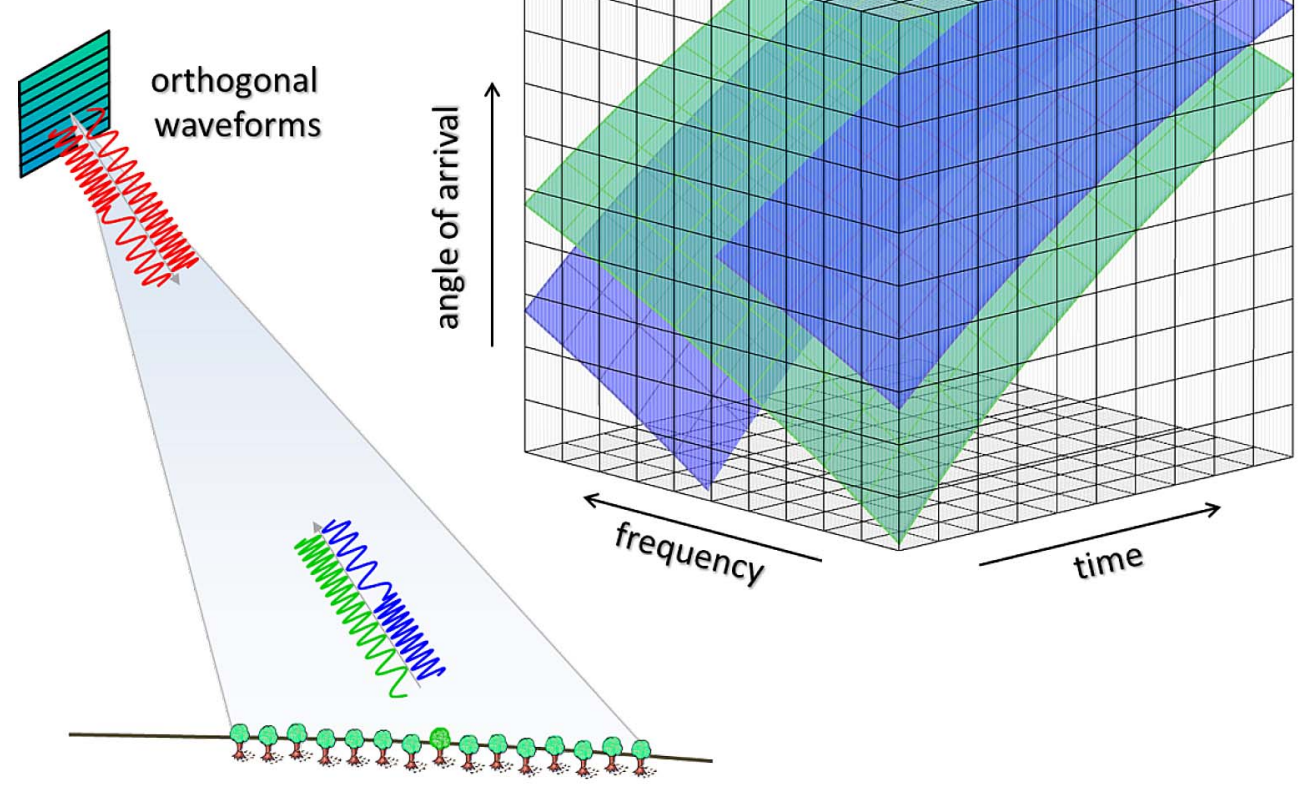

Fig. 14. Information cube in case of the simultaneous reception of two orthogonal waveforms received from a distributed scattering scenario. The receiving radar antenna has multiple elevation channels which allow a spatial filtering of the incoming signal according to its angle of arrival. This is indicated on the right-hand side by additional grid lines which divide the information cube in the vertical direction.

received, which are mutually displaced in both time and angle of arrival. In the limit of a distributed scatterer scenario with a large number of individual scatterers (e.g., dense forest), the green surface will be obtained. The surface therefore provides a succinct illustration of the intimate coupling between range time, instantaneous frequency, and angle of arrival for a sidelooking imaging radar operated with chirped transmit signals.

A different picture arises if multiple orthogonal signals are transmitted at the same time. As an illustrative example, let us consider the waveform from Fig. 11. Assuming again first a scene composed of a single point-like scatterer on the ground, the information cube shown in Fig. 13 is obtained. It is clear that the orthogonal signals have no overlap in the time-frequency plane. It is therefore no problem to separate the orthogonal returns from the single point scatterer by an appropriate time-frequency processing.

A more complicated picture arises if the scene is composed of multiple distributed scatterers (forest scenario). In this case, each time-frequency cell of the cube becomes filled by two vertically overlapping surfaces, as shown in Fig. 14. It is therefore impossible to separate the signal returns from the two simultaneously transmitted waveforms in the time-frequency plane. A separation becomes, however, possible if additional information on the spatial direction of the arriving radar echoes is provided. This extra information is illustrated in Fig. 14 by the additional horizontal grid lines which divide the cube in the vertical direction. The vertical spacing between the grid lines indicates the radar's ability to separate signals from different angles of arrival and depends essentially on the antenna height and the associated number of receiver channels. From Fig. 14, it can also be seen that the surfaces approach each other in the vertical direction as the radar echo time $t$ increases. Larger echo times correspond to more distant scatterers mapped with increasing incident angles. Hence, a more extended antenna will be required to separate the signal echoes from large incident angles. An operation at lower incident angles is therefore preferred in order to keep the antenna size as small as possible. It should, moreover, be clear from Fig. 14 that the orthogonal waveforms should be designed such that the instantaneous frequency separation is maximized. In this sense, the heuristically chosen waveforms from Fig. 11 can already be regarded as a good choice.

Another interesting aspect arises from the visualization by means of the information cube. Taking again a look at Fig. 12, it seems that each independent time-frequency cell of a singlechannel SAR system without any beamforming capabilities in elevation is already filled with information. There is hence no space left to accommodate in the received signal the additional information that might be provided by the transmission of other waveforms, independent whether they are orthogonal or not. This limitation can also be understood in a more abstract mathematical sense by regarding the SAR data acquisition and focusing processes as mappings between object, data, and image spaces [26]. The object space means here essentially the set of all possible scene reflectivity functions (including white noise scenes), the data space represents the raw data collected by the radar, and the image space corresponds to the set of focused SAR images reconstructing the scene reflectivity within the radar's limitations. To simplify the discussion, we assume in the following a one-to-one (i.e., bijective) mapping between the data space and the image space, i.e., the dimensions of the data and image spaces are equal, and one element of the 
SAR Data Acquisition

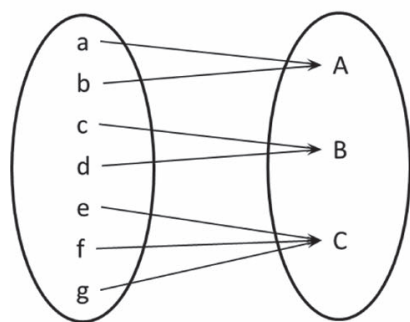

Object Space
Data Space $\underline{\text { Scene Reconstruction }}$

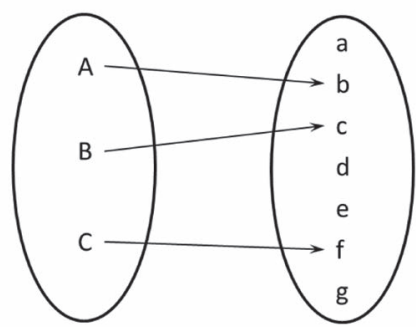

Data Space
Object Space
Fig. 15. Mapping from object space to data space and back in case of a singlechannel receiver.
Multi-ChannelSAR

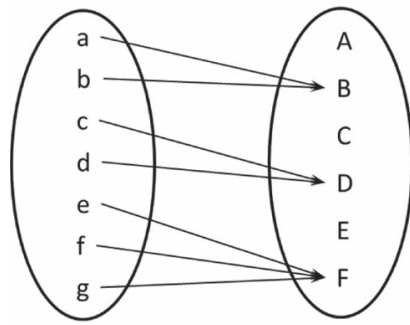

Object Space
Data Space

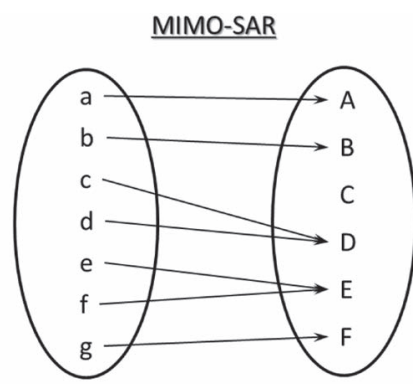

Object Space
Data Space
Fig. 16. Mapping from object space to data space for a receiver with multiple elevation channels (left) and a MIMO-SAR with appropriately designed Tx waveforms.

image space corresponds to exactly one element of the raw data space and vice versa. This means that we can take the data and image spaces as synonymous. To concentrate on the essential aspects, we further neglect any effects from oversampling, noise, and saturation during the raw data acquisition. Under this assumption, the data acquisition of a well-designed SAR system can essentially be understood as a surjective (i.e., onto) mapping from the object space to the data (or image) space (cf., Fig. 15, left). This means that many elements from the object space are mapped to a single element of the data space. The many-to-one mapping is, besides other things, caused by the resolution limitations of the radar. On the other hand, at least one possible element within the object space corresponds to each element of the data space. ${ }^{6}$ There exists therefore an injective (one-to-one) mapping from the data space to the object space (cf., Fig. 15, right). This means that each element of the data space corresponds to a distinct scene, as long as the set of possible scenes is not restricted a priori by specific regularity assumptions. The transmission of additional (e.g., orthogonal) waveforms will therefore inevitably change the received signal in the data space and, therefore, also the assigned element in the object (and image) space.

A different picture arises if the received radar signal is sampled by multiple elevation channels. In this case, the mapping from the object space to the data space is no longer surjective, as illustrated in Fig. 16 on the left. A MIMO-SAR can therefore use the free elements in the data space to provide further

\footnotetext{
${ }^{6}$ This assumes, as mentioned before, that there exists no oversampling in the raw data, i.e., the data space does not contain redundancies.
}

information about the scene by transmitting additional radar pulses with appropriately designed Tx waveforms. This was, for example, exploited in the technique of multidimensional waveform encoding to reduce the ambiguities of a spaceborne SAR system by transmitting dedicated space-time-coded signals, thereby assigning new information about the object space to the free elements of the data space [2]. The underlying principle is illustrated on the right-hand side of Fig. 16 where, for example, the elements $a$ and $b$ of the object space are now mapped to different elements $A$ and $B$ in the data space and can therefore be separated from each other. Appropriately designed MIMO-SAR waveforms should hence try to take advantage of the additional degrees of freedom that can be provided by a multichannel receiver. With reference to the framework of information theory, this corresponds to an increase of the entropy within the data space which directly reflects the additional information provided about the different elements in the object space. On the other hand, it seems futile to gather additional information about the object space if the data space is already completely filled with information, i.e., if it has reached maximum entropy. This applies, for example, in good approximation to the case of a single-channel SAR which images a distributed scene with arbitrary scatterer arrangements (white noise scene).

The previous reasoning implicitly assumed that the scatterer distribution can be regarded as (higher order) white noise, and one may argue that the SAR data acquired from real-world scenes are full of second- and higher-order correlations, as it is the case for optical images [30], [31]. ${ }^{7}$ To exploit these redundancies, it would, however, first be necessary to identify those regions in the data space which are not already filled with information. This unused space could then either be exploited for data compression or for MIMO-SAR imaging. Simple examples for such an approach are the imaging of short scenes by long waveforms or the mapping of a scene composed of a set of sparsely distributed scatterers (ships on the sea scenario) as discussed in Section III. In both cases, the data space is only sparsely filled with information. It should, however, be clear from the previous analyses that the mere use of orthogonal waveforms without any explicit consideration of such redundancies in the data space seems to promise little success for the successful implementation of MIMO-SAR systems. Even in the case where one can identify redundancies within the data space (which are presumably more prominent in the image space after SAR focusing), it remains an open question whether appropriate $\mathrm{Tx}$ waveforms can be found, which exploit the unused subspace for MIMO-SAR imaging purposes. This complication is a result from the intricate data acquisition process of SAR systems which disperse the scattered signal from individual objects over many raw data samples.

\section{OFDM: An ALternative?}

Recently, several new SAR imaging techniques have been studied, which replace the commonly used linear frequency

\footnotetext{
${ }^{7}$ On the other hand, one should keep in mind that, for example, mediumresolution-focused SAR images from a homogeneous undisturbed rain forest look similar to white noise, and one may have even difficulties to differentiate them from images derived via a mere SAR focusing of white noise.
} 


\section{Chirp Generation}

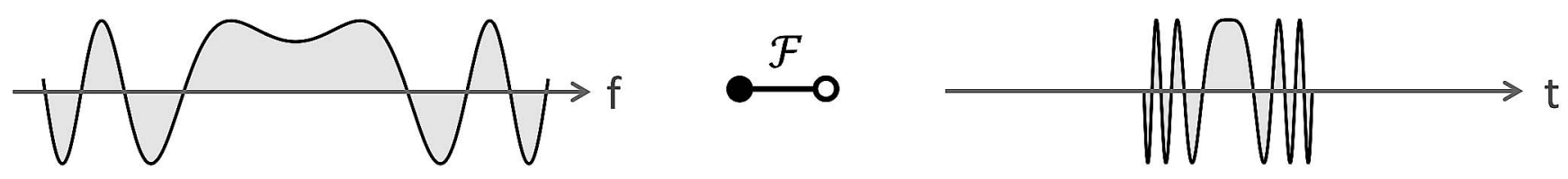

$\underline{\text { OFDM Modulation }}$

$*$
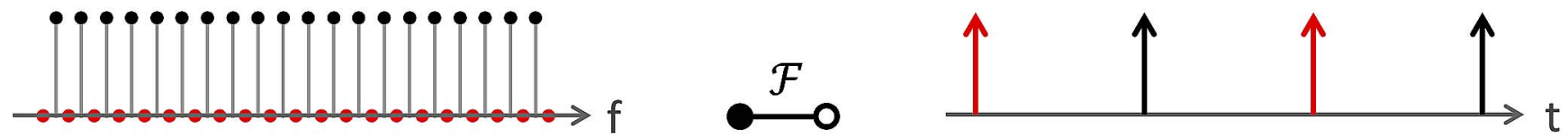

* $\quad$ D/A Conversion
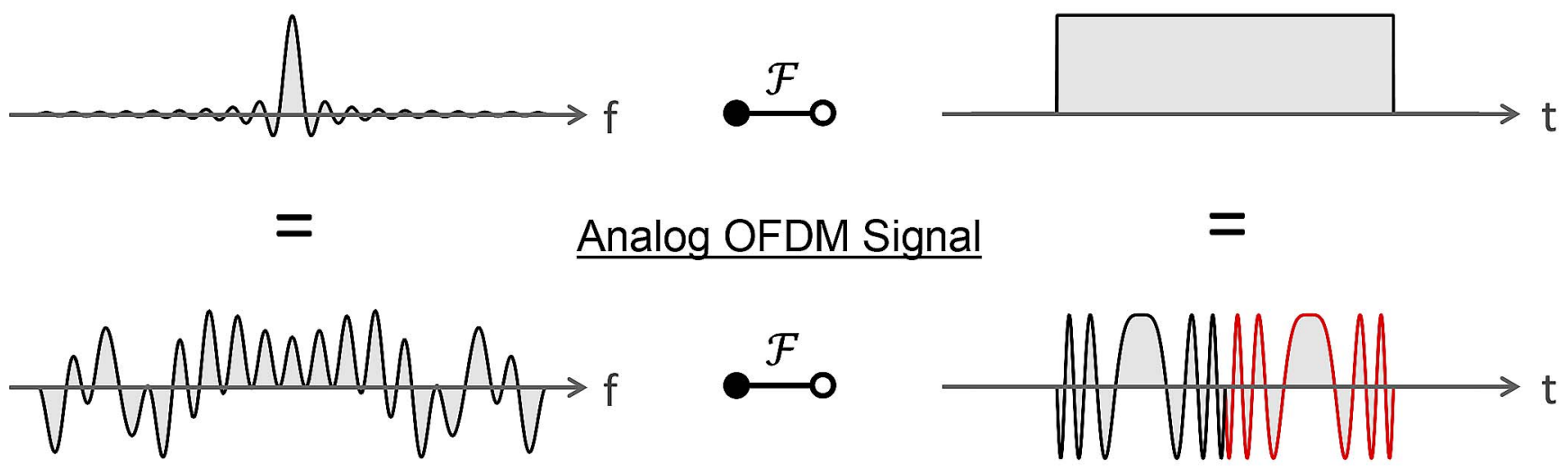

Fig. 17. OFDM waveform generation in the frequency (left) and time (right) domains. A conventional chirped signal is sampled in the frequency domain by a sequence of Dirac pulses which represent the OFDM carriers. This multiplication corresponds in the continuous time domain to a convolution of a chirped signal with a sequence of Dirac pulses. The proposed suppression of every second frequency component (red dots) increases the effective spacing between the sample positions in the frequency domain and therefore decreases the corresponding Dirac spacing in the time domain by a factor of two as indicated by the additional red arrows. This implies, besides the original signal repetition from the sampling in the frequency domain, an additional repetition of the chirped signal in the time domain. The transition from the signal representation in the discrete frequency domain to a continuous frequency domain representation implies a convolution with a sinc function. This corresponds in the time domain to a multiplication by a rectangular window. The final result is the desired OFDM waveform shown at the lower right, which consists in the time domain essentially of two repeated chirped pulses as suggested in [16]. The second and orthogonal waveform is

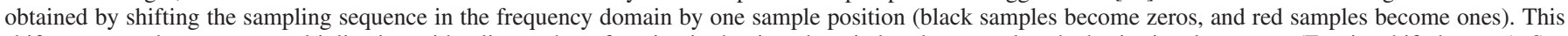
shift corresponds to a mere multiplication with a linear phase function in the time domain but does not alter the basic signal structure (Fourier shift theorem). See [33] for a more detailed derivation.

modulated (LFM) chirp pulses of conventional SAR systems by a novel class of transmit waveforms that are based on OFDM techniques [16], [17], [20], [27]-[29], [33]. To obtain a pair of orthogonal transmit signals suitable for MIMO-SAR imaging, it was further suggested in [16], [29], and [33] to divide the available bandwidth of two discrete and time-limited chirp signals in the frequency domain by assigning subsequent frequency components to either one or the other waveform. This results in the discrete frequency domain representation in a comblike spectrum for each Tx signal and, therefore, no spectral overlap between the two waveforms (cf., Fig. 17). Coherence between the otherwise uncorrelated SAR images obtained from this pair of orthogonal waveforms is then retained by limiting the scene extension to less than half of the length of the transmitted OFDM waveforms. This requires the partitioning of a wide image swath into multiple small subswaths by using a multichannel receiver that is capable of generating multiple narrow antenna beams in elevation.

To better understand the implications of the special kind of OFDM SAR data acquisition suggested in [16], [29], and [33], it is instructive to take a closer look at the transmitted waveforms. Fig. 17 shows that the discrete comb-like OFDM spectrum corresponds in the continuous time domain to a signal that is composed of two repeated subpulses. The pulse repetition is independent of the original waveform and an immediate consequence of the comb-like spectral sampling where each second frequency component is set to zero. The repeated subpulse structure leads inevitably to additional peaks in the autocorrelation function of the proposed OFDM waveform. This implies, in turn, that the focused image from each 


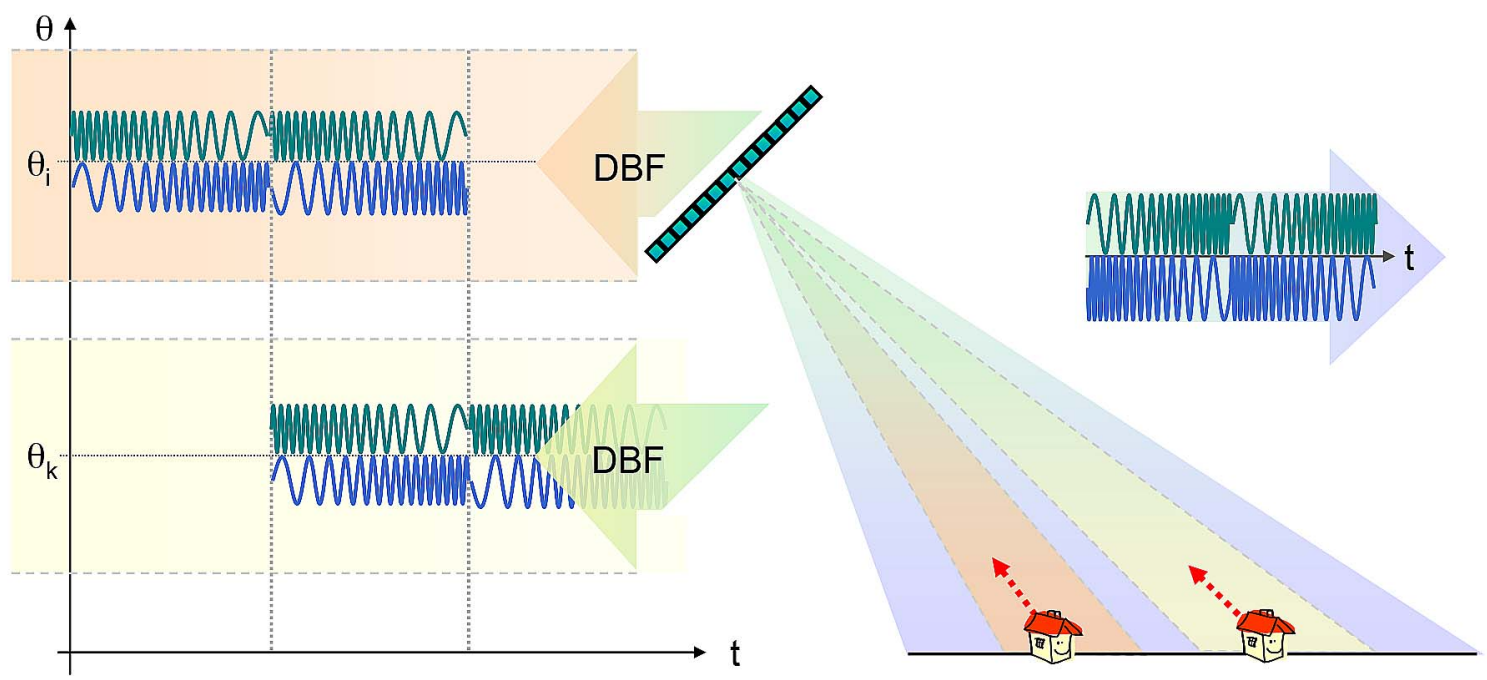

Fig. 18. Use of OFDM signals results in range ambiguities that must be resolved by DBF on receive.

individual waveform becomes ambiguous in range as soon as the scene extension is longer than half the OFDM pulse length. The mere use of OFDM transmit signals together with an appropriate matched filter (or any other OFDM processing) is therefore not sufficient for an unambiguous wide-swath MIMOSAR imaging!

The range ambiguity from the proposed OFDM signals can only be resolved by providing additional information on the direction of the arriving wavefronts. This requires, in turn, a sufficiently extended antenna array with multiple elevation channels. The extra information from the multichannel receiver can then be used to suppress the range ambiguity by DBF on receive as illustrated in Fig. 18. It should hence be clear that the suggested OFDM-MIMO-SAR employs the same basic principle as it was originally suggested for the concept of multidimensional waveform encoding (cf., [2] and Section IV). The minimum antenna height is again provided by (5), where $\Delta \tau$ corresponds now to half of the duration of the transmitted OFDM pulse. Note that this requirement on the antenna height is equal to that for the mutual suppression of the previously suggested short-term shift-orthogonal waveforms from Fig. 11. Only for scenes that are shorter than half of the transmitted pulse length, one may neglect the range ambiguity suppression by DBF on receive. This may, for example, be a viable solution in a bistatic automotive radar [32] or the ISAR system from [19] where the range from which radar echoes are received above noise level is much shorter than the length of the transmitted pulse.

After pointing out that MIMO-SAR imaging with OFDM and multidimensional waveform encoding are based on the same underlying principle, the question remains which kind of waveform encoding is the most appropriate. For this, one should be aware that, in the suggested OFDM modulation, a possible issue arises from the close separation of the frequency components which are assigned to either one or the other waveform. If, for example, a 100- $\mu$ s long radar pulse is employed, the individual OFDM frequency components (or carriers) will be separated by only $10 \mathrm{kHz}$. Such a separation is, however, already close to the Doppler bandwidth of a spaceborne high-resolution wide-swath SAR system with 1-m azimuth resolution. ${ }^{8}$ It is therefore inevitable that a notable portion of the Doppler spectrum is leaked into the "orthogonal" channel, thereby deteriorating the image quality. In contrast, the instantaneous frequencies of the waveforms from Fig. 11 are typically separated by several $10 \mathrm{MHz}$ or even $100 \mathrm{MHz}$, and Doppler leakage is therefore not an issue at all. Another problem may arise from the fact that the OFDM processing suggested in [33] requires, before range focusing, a complete suppression of all signals that arise from scatterers outside the processing interval. This implies a rectangular window which may be difficult to implement in a practical system. On the other hand, the short-term shift-orthogonal waveforms from Fig. 11 allow the elevation beamforming to be performed either before or after the range processing, and the interferences from the short-term shift-orthogonal waveforms can even be suppressed for each individual range bin by a highly efficient null steering of the antenna pattern.

\section{FuRTher OpPortunities AND OUtLOOK}

MIMO-SAR with appropriately designed transmit waveforms enables in the long term not only the implementation of fully polarimetric high-resolution wide-swath SAR systems or the acquisition of additional phase centers for novel SAR applications like multibaseline interferometry or tomography but also a completely new class of hybrid and adaptive SAR imaging modes [1], [2]. These novel modes can, for example, be used to provide at the same time different spatial or radiometric resolutions for different image regions. This opportunity may be of great benefit in the design of future SAR missions that try to resolve hitherto incompatible user requirements. As a simple example, one may consider a SAR mission like Sentinel-1 that

\footnotetext{
${ }^{8}$ The minimum Doppler bandwidth of a SAR can be approximated by $B_{\text {Dop }}=v_{g} / \Delta a z$, where $v_{g}$ is the velocity of the satellite's antenna beam on the ground and $\Delta a z$ is the azimuth resolution. A typical spaceborne SAR system in a low Earth orbit has a beam ground velocity of $v_{g}=7000 \mathrm{~m} / \mathrm{s}$, which leads, for an azimuth resolution of $\Delta a z=1 \mathrm{~m}$, to a Doppler bandwidth of $B_{\text {Dop }}=7000 \mathrm{~Hz}$.
} 
systematically maps the whole Earth surface with a moderate resolution of $20 \mathrm{~m}$ in a ScanSAR-like mode every 12 days. A hybrid MIMO-SAR mode could then allow for the simultaneous acquisition of an additional narrow stripmap swath with a significantly improved spatial resolution [1]. By this, one could offer the user community both a coarse resolution SAR image of the Earth every 12 days and a fine resolution image of the whole Earth surface every one or two months without a too significant increase in both the downlink and the power budgets. Hybrid and adaptive MIMO-SAR modes may also be of high interest for advanced concepts that employ SAR satellites in a Medium Earth (MEO) or even geosynchronous orbit (GEO). ${ }^{9}$ The increased orbital altitude can provide a huge instantaneous field of view that typically extends across several thousand kilometers. High-altitude systems provide, moreover, the opportunity to continuously observe a given area for a long time period ranging from several minutes up to hours. This may, for example, be exploited for advanced traffic monitoring concepts as shortly discussed in [45]. For this, new data acquisition strategies based on hybrid and adaptive MIMO-SAR modes will be desired to stay within both the available power and downlink budget. The new short-term shift-orthogonal waveforms discussed in this paper are an enabling technology for many of these advanced MIMO-SAR techniques and open the door for future SAR missions with hitherto unknown imaging and mapping capabilities.

\section{CONCLUSION}

MIMO-SAR offers the opportunity to improve the imaging performance of future SAR systems and to acquire novel interferometric and tomographic information products with a minimum number of antenna elements. For this, multiple transmit signals have to be employed, and it has to be ensured that the radar echoes from each transmit channel can be extracted without any interferences that may arise from the scene illumination by the other transmit channels. In selected airborne scenarios, it may be sufficient to transmit the different Tx signals in subsequent PRIs [6], [7], [11], [12]. Such a strategy is, however, in general, not applicable to a spaceborne scenario where the high

\footnotetext{
${ }^{9}$ One reviewer asked whether such a MEO- or even GEO-MIMO-SAR system is realistic in terms of the required antenna height. For this, we may consider an exemplary X-band MEO-SAR system with an orbit height of $10000 \mathrm{~km}$. The instantaneous Doppler bandwidth acquired by such a system depends on the selected orbit, particularly whether a retrograde or a prograde orbit is chosen. Assuming an antenna length of $10 \mathrm{~m}$, the Doppler bandwidth will be below $1000 \mathrm{~Hz}$ in the latter case. It is, moreover, important to note in this context that the azimuth resolution depends not on the satellite velocity but on the antenna footprint velocity. In consequence, an azimuth resolution of $2 \mathrm{~m}$ can be achieved in stripmap mode in this example despite the use of a 10 -m-long antenna. Assuming in (5) a PRF of $1 \mathrm{kHz}$ and a $\Delta \tau$ of $150 \mu \mathrm{s}$, the required antenna heights are approximately 10 and $15 \mathrm{~m}$ for incident angles of $30^{\circ}$ and $45^{\circ}$, respectively. The required aperture could, for example, be provided by a suitably designed reflect array or a large deployable reflector antenna in combination with advanced DBF techniques as already suggested for Tandem-L [1]. Note, moreover, that the antenna size required for ambiguity and mutual subpulse suppression decreases as the angular velocity of the satellite approaches the angular velocity of the Earth, as it is the case for a geosynchronous SAR. We therefore consider MEO- and GEO-MIMO-SAR systems as realistic options for the future, as long as the range resolution requirements do not ask for a large bandwidth which would then be associated with a low signal-to-noise ratio (SNR).
}

sensor velocity poses notable ambiguity constraints. A prominent example for this fundamental limitation is the reduced unambiguous swath width in the alternating bistatic mode of TanDEM-X which can be regarded as a MIMO-SAR with two transmit and two receive channels that provide together three virtual phase centers [34]. ${ }^{10}$ A reliable separation of the radar echoes arising from the simultaneous use of multiple transmit signals, i.e., a signal extraction free of mutual interferences, is therefore a fundamental challenge for the implementation of future spaceborne MIMO-SAR systems. To overcome this fundamental MIMO-SAR challenge, it was suggested within this paper to simultaneously transmit multiple waveforms that are mutually short-term shift orthogonal. The short-term shift orthogonality ensures a reliable signal separation without mutual interference for closely spaced scatterers. Interferences from the lack of orthogonality for longer mutual shifts are then suppressed by DBF on receive in elevation. By this, the special data acquisition geometry of a side-looking imaging radar is exploited. The signal separation from the multiple transmit waveforms requires a large antenna height if compared to conventional SAR systems. Antennas with increased heights and multiple receive channels have already been suggested to improve the sensitivity of future SAR systems [35]-[39] where the associated real-time beamsteering in elevation is also known as scan-on-receive. The spatial waveform separation in elevation may, in the future, greatly benefit from the more recently suggested SAR architectures that combine a large unfoldable reflector antenna with a digital feed array [1], [40]-[44]. The rather large antenna height (if compared to conventional spaceborne SAR systems) provides ideal conditions to separate the radar echoes from the short-term shift-orthogonal waveforms. First ideas to use reflector antennas for MIMO-SAR imaging purposes have already been discussed in [46].

The concept of the information cube provided further insights into the peculiarities of single and multichannel SAR data acquisitions, and it became evident that the signal space provided by a multichannel receiver system is only sparsely filled with information. MIMO-SAR systems can therefore exploit the additional degrees of freedom provided by a multichannel receiver. For this, the vacant cells of the information cube are to be filled with extra information about the imaged scene without destroying the information in the already occupied cells. This can be accomplished by transmitting suitably designed waveforms either from different antennas or with different polarizations. The suggested short-term shift-orthogonal waveforms represent one class of suitable signal modulations that will serve this purpose. As an alternative, a special kind of OFDM waveforms has been suggested in [33] for the same purpose of MIMO-SAR imaging. An analysis of these two alternative waveforms revealed that, in both cases, the additional information from a multichannel receiver is employed to suppress either the interference from the orthogonal channel (in case of short-term shift-orthogonal waveforms) or the

\footnotetext{
${ }^{10}$ If the TerraSAR-X and TanDEM-X satellites are each operated in the dual-receive antenna mode, a MIMO-SAR with two transmit and four receiver channels can be formed [34]. This results in a MIMO-SAR with eight virtual phase centers, where two out of these eight phase centers are almost redundant.
} 
ambiguity from the repeated subpulse structure (in case of OFDM). A first comparison between these alternative waveforms shows some advantages for the short-term shiftorthogonal waveforms of Fig. 11, but this topic may deserve further investigation in the future.

\section{APPENDIX A}

As suggested by one reviewer, we provide here a more rigorous analytical derivation of the results from Section III-A. For this, we assume a radar that simultaneously transmits the two waveforms $s_{i}(t)$ and $s_{j}(t)$. The received radar echo from a point scatterer located at distance $r$ will then be given by

$$
s(t)=s_{i}\left(t-\frac{2 r}{c}\right)+s_{j}\left(t-\frac{2 r}{c}\right)
$$

where $c$ denotes the speed of wave propagation. For convenience, any unimportant amplitude scaling due to free space loss, antenna gain, RCS, etc., has been omitted in (8). The range-compressed signal with reference to the first transmit channel is obtained by matched filtering $s(t)$ with $s_{i}^{*}(-t)$. Taking into account the linearity of the matched filtering operation, this yields

$$
\begin{aligned}
u(t)= & s(t) * s_{i}^{*}(-t) \\
= & \int s_{i}\left(\tau-\frac{2 r}{c}\right) \cdot s_{i}^{*}(\tau-t) \cdot d \tau \\
& +\int s_{j}\left(\tau-\frac{2 r}{c}\right) \cdot s_{i}^{*}(\tau-t) \cdot d \tau
\end{aligned}
$$

where $*$ denotes the linear convolution operator. The first integral on the right-hand side of (9) describes the expected rangefocused point scatterer response if only the waveform $s_{i}(t)$ had been transmitted, while the second integral corresponds to an additional contribution in the range-focused signal. This undesired term is due to the simultaneous transmission of waveform $s_{j}(t)$. In the following, we denote this second term in (9) as $u_{x}(t)$

$$
u_{x}(t)=\int s_{j}\left(\tau-\frac{2 r}{c}\right) \cdot s_{i}^{*}(\tau-t) \cdot d \tau .
$$

The integrated energy of this undesired point target response can be written as

$$
\left\langle\left|u_{x}(t)\right|^{2}\right\rangle=\int u_{x}(t) \cdot u_{x}^{*}(t) \cdot d t
$$

Using Plancherel's (commonly denoted as Parseval's) theorem, one obtains in the frequency domain an equivalent expression for the range processed signal energy

$$
\left\langle\left|u_{x}(t)\right|^{2}\right\rangle=\int U_{x}(f) \cdot U_{x}^{*}(f) \cdot d f
$$

where $U_{x}(f)$ denotes the Fourier transform of $u_{x}(t)$. The spectrum $U_{x}(f)$ can be derived from (10) by employing both the convolution and the Fourier shift theorem as

$$
U_{x}(f)=S_{i}^{*}(f) \cdot S_{j}(f) \cdot \exp \left[-j \frac{4 \pi r}{c} f\right]
$$

where $S_{i}(f)$ and $S_{j}(f)$ denote the Fourier transforms of $s_{i}(t)$ and $s_{j}(t)$, respectively. Substituting this equation in (12), we obtain for the energy of the range processed signal

$$
\left\langle\left|u_{x}(t)\right|^{2}\right\rangle=\int\left|S_{i}(f)\right|^{2} \cdot\left|S_{j}(f)\right|^{2} \cdot d f .
$$

From this equation, it should be immediately apparent that any overlap between the two spectra $S_{i}(f)$ and $S_{j}(f)$ causes inevitably a nonvanishing energy contribution from the unmatched channel in the focused image. For the most interesting case where both $S_{i}(f)$ and $S_{j}(f)$ have flat spectra of equal magnitude, one can easily compute the SNR as

$$
\mathrm{SNR}=\frac{\left\langle\left|u_{m}(t)\right|^{2}\right\rangle}{\left\langle\left|u_{x}(t)\right|^{2}\right\rangle}=\frac{\int\left|S_{i}(f)\right|^{2} \cdot\left|S_{i}(f)\right|^{2} \cdot d f}{\int\left|S_{i}(f)\right|^{2} \cdot\left|S_{j}(f)\right|^{2} \cdot d f}=1
$$

where we denoted the first term of the right-hand side of (9) with the "matched" response $u_{m}(t)$. It is therefore evident that the SAR image will suffer from a considerable energy leakage from the orthogonal transmit signal. In case that more than two orthogonal signals are transmitted at the same time, the "noise" contributions will add, and it is straightforward to show that the SNR will, in the case of equal magnitude spectra, further decrease to $1 /(N-1)$, where $N$ denotes the number of simultaneously transmitted waveforms. The only means to get rid of the noise contributions from the orthogonal channels is an additional filtering, as outlined in Section IV.

\section{APPENDIX B}

In this appendix, we derive a pair of orthogonal waveforms that satisfy the orthogonality condition in (1). For this, we start with the two LFM waveforms (chirps)

$$
\begin{aligned}
& s_{1}(t)=\operatorname{rect}\left(\frac{t}{T_{p}}\right) \cdot \exp \left(-j \pi k_{r} t^{2}\right) \\
& s_{2}(t)=\operatorname{rect}\left(\frac{t}{T_{p}}\right) \cdot \exp \left(j \pi k_{r} t^{2}\right)
\end{aligned}
$$

where $k_{r}=B_{r} / T_{p}$ is the chirp rate defined as the ratio between the chirp bandwidth $B_{r}$ and the chirp duration $T_{p}$. The evaluation of the cross-correlation integral (1) then yields

$$
\begin{aligned}
\int_{-T_{p} / 2}^{T_{p} / 2} s_{1}^{*}(t) \cdot s_{2}(t) \cdot d t & =\int_{-T_{p} / 2}^{T_{p} / 2} \exp \left(j \pi k_{r} t^{2}\right) \cdot \exp \left(j \pi k_{r} t^{2}\right) \cdot d t \\
& =\int_{-T_{p} / 2}^{T_{p} / 2} \exp \left(j 2 \pi k_{r} t^{2}\right) \cdot d t \\
& =\frac{e^{-j \frac{3 \pi}{4}}}{\sqrt{2 k_{r}}} \cdot \operatorname{Erf}\left[\frac{j-1}{2} T_{p} \sqrt{\pi k_{r}}\right]
\end{aligned}
$$

where Erf is the Gaussian error function. It is clear that the upand down-chirps do not fulfill the orthogonality condition in (1). We can, however, easily cure this lack of orthogonality by 
subtracting a "homeopathic" term $s_{\Delta}(t)$ from the second chirp signal

$$
s_{\perp}(t)=s_{2}(t)-s_{\Delta}(t) .
$$

In this case, we obtain for the correlation integral in (1) the expression

$$
\begin{aligned}
& \int_{-T_{p} / 2}^{T_{p} / 2} s_{1}^{*}(t) \cdot s_{\perp}(t) \cdot d t \\
& =\int_{-T_{p} / 2}^{T_{p} / 2} s_{1}^{*}(t) \cdot s_{2}(t) \cdot d t-\int_{-T_{p} / 2}^{T_{p} / 2} s_{1}^{*}(t) \cdot s_{\Delta}(t) \cdot d t \\
& =\frac{e^{-j \frac{3 \pi}{4}}}{\sqrt{2 k_{r}}} \cdot \operatorname{Erf}\left[\frac{j-1}{2} T_{p} \sqrt{\pi k_{r}}\right] \\
& \quad-\int_{-T_{p} / 2}^{T_{p} / 2} \exp \left(j \pi k t^{2}\right) \cdot s_{\Delta}(t) \cdot d t
\end{aligned}
$$

There is a great degree of freedom in choosing an appropriate function $s_{\Delta}(t)$ such that the aforementioned correlation integral vanishes. A particularly simple solution arises if we chose

$$
s_{\Delta}(t)=\alpha \cdot \operatorname{rect}\left(\frac{t}{T_{p}}\right) \cdot \exp \left(-j \pi k t^{2}\right)
$$

where $\alpha$ is a constant to be immediately determined. Substituting (21) into (20), the evaluation of the last integral in (20) becomes trivial, and we can ensure a vanishing crosscorrelation by simply choosing the constant $\alpha$ as

$$
\alpha=\frac{e^{-j \frac{3 \pi}{4}}}{T_{p} \sqrt{2 k_{r}}} \cdot \operatorname{Erf}\left[\frac{j-1}{2} T_{p} \sqrt{\pi k_{r}}\right] .
$$

Assuming, for example, a bandwidth of $B_{r}=100 \mathrm{MHz}$ and a pulse length of $T_{p}=50 \mu \mathrm{s}$, which may both be regarded as typical parameters for a spaceborne SAR system like TanDEM-X, we obtain for $\alpha$ a value of $\alpha=0.00707+$ $j 0.00701$. The magnitude of the correction signal $s_{\Delta}(t)$ is hence less than $1 \%$ of the magnitude of the original signal $s_{2}(t)$. From this, it should be clear that the slight modification has only a small effect on the MIMO-SAR imaging performance. This has also been confirmed numerically by conducting the simulations in Figs. 4 and 5 twice, once using $s_{\perp}(t)$ of (19) and once using $s_{2}(t)$ of (17) for the second waveform.

\section{ACKNOWLEDGMENT}

The author would like to thank both the anonymous reviewers and the editors for their valuable comments and suggestions that helped in improving the clarity of this paper. The author also expresses his gratitude to his colleagues P. Lopez Dekker, J.-H. Kim, M. Younis, F. de Zan, and A. Moreira for the many helpful discussions on MIMO-SAR in general and OFDM in particular. It turned out during both these discussions and from the many citations in the literature that the former concept of multidimensional waveform encoding had, by numerous authors, not been understood as a MIMO-SAR technique. This was one of the motivations to present and extend this old concept in a different and more general form which relates and compares it explicitly to present MIMO-SAR publications. The author would also like to thank Prof. W. Wiesbeck for the continued effort to advance the use of new radar waveforms and MIMO techniques for the purpose of improved SAR imaging. It was in the end his merit that this paper had been written. The hardware for a modular MIMO radar demonstrator to test the ideas and concepts put forward in this and many related papers is currently being developed at DLR by T. Rommel, A. Patyuchenko, and P. Laskowski.

\section{REFERENCES}

[1] G. Krieger, I. Hajnsek, K. Papathanassiou, M. Younis, and A. Moreira, "Interferometric synthetic aperture radar (SAR) missions employing formation flying," Proc. IEEE, vol. 98, no. 5, pp. 816-843, May 2010.

[2] G. Krieger, N. Gebert, and A. Moreira, "Multidimensional waveform encoding: A new digital beamforming technique for synthetic aperture radar remote sensing," IEEE Trans. Geosci. Remote Sens., vol. 46, no. 1, pp. 31-46, Jan. 2008.

[3] R. C. Heimiller, J. E. Belyea, and P. G. Tomlinson, "Distributed array radar," IEEE Trans. Aerosp. Electron. Syst., vol. AES-19, no. 6, pp. 831839, Nov. 1983

[4] E. Fishler, A. Haimovich, R. Blum, D. Chizhik, L. Cimini, and R. Valenzuela, "MIMO radar: An idea whose time has come," in Proc. IEEE Radar Conf., Philadelphia, PA, USA, Apr. 2004, pp. 71-78.

[5] J. Li and P. Stoica, MIMO Radar Signal Processing. Hoboken, NJ, USA: Wiley, 2009.

[6] G. Krieger, J. Mittermayer, S. Buckreu, M. Wendler, T. Sutor, F. Witte, and A. Moreira, "Sector imaging radar for enhanced vision," Aerosp. Sci. Technol., vol. 7, no. 2, pp. 147-158, Mar. 2003.

[7] J. Klare, A. Brenner, and J. Ender, "A new airborne radar for 3D imaging-Image formation using the ARTINO principle," presented at the Eur. Conf. Synthetic Aperture Radar (EUSAR), Dresden, Germany, 2006.

[8] G. Krieger, N. Gebert, and A. Moreira, "Hochauflösendes SynthetikApertur-Seitensicht-Radarsystem mittels digital beamforming (highresolution synthetic aperture side view radar system used by means of digital beamforming)," Patent EP 2018577 B1, May 4, 2007.

[9] G. Krieger, N. Gebert, and A. Moreira, "Digital beamforming techniques for spaceborne radar remote sensing," presented at the Eur. Conf. Synthetic Aperture Radar (EUSAR), Dresden, Germany, 2006.

[10] A. Currie and M. A. Brown, "Wide-swath SAR," Proc. Inst. Elect. Eng.-Radar Signal Process., vol. 139, no. 2, pp. 122-135, Apr. 1992.

[11] J. Ender, "MIMO-SAR," in Proc. Int. Radar Symp., Cologne, Germany, 2007, pp. 667-674.

[12] D. Christallini, D. Patina, and P. Lombardo, "Exploiting MIMO SAR potentialities with efficient cross-track constellation configurations for improved range resolution," IEEE Trans. Geosci. Remote Sens., vol. 49, no. 1, pp. 38-52, Jan. 2011.

[13] C. Prati and F. Rocca, "Improving slant-range resolution with multiple SAR surveys," IEEE Trans. Aerosp. Electron. Syst., vol. 29, no. 1, pp. 135-143, Jan. 1993.

[14] A. Freeman, W. T. K. Johnson, B. Huneycutt, R. Jordan, S. Hensley, P. Siqueira, and J. Curlander, "The 'myth' of the minimum SAR antenna area constraint," IEEE Trans. Geosci. Remote Sens., vol. 38, no. 1, pp. 320-324, Jan. 2000.

[15] G. Krieger, N. Gebert, and A. Moreira, "Multidimensional waveform encoding for synthetic aperture radar remote sensing," in Proc. IET Int. Conf. Radar Syst., Edinburgh, U.K., 2007, pp. 1-5.

[16] J. Kim, M. Younis, A. Moreira, and W. Wiesbeck, "A novel OFDM waveform for fully polarimetric SAR data acquisition," in Proc. EUSAR, Aachen, Germany, 2010, pp. 1-4.

[17] W.-Q. Wang, "Space-time coding MIMO-OFDM SAR for high-resolution imaging," IEEE Trans. Geosci. Remote Sens., vol. 49, no. 8, pp. 30943104, Aug. 2011.

[18] W.-Q. Wang, "Virtual antenna array analysis for MIMO synthetic aperture radars," Int. J. Antennas Propag., vol. 2012, p. 587 276, 2012. 
[19] G. Castellanos, M. Jirousek, and M. Peichl, "Orthogonal waveform experiments with a highly digitized radar," in Proc. EUSAR, Nuremberg, Germany, 2012, pp. 103-106.

[20] W.-Q. Wang, "Mitigating range ambiguities in high-PRF SAR with OFDM waveform diversity," IEEE Geosci. Remote Sens. Lett., vol. 10, no. 1, pp. 101-105, Jan. 2013

[21] W.-Q. Wang, "Applications of MIMO technique for aerospace remote sensing," in Proc. IEEE Aerosp. Conf., 2007, pp. 1-10.

[22] W.-Q. Wang and J. Cai, "Ground moving target indication by MIMO SAR with multi-antenna in azimuth," in Proc. IEEE IGARSS, Vancouver, BC, Canada, 2011, pp. 1662-1665.

[23] J. Klare, "Digital beamforming for a 3D MIMO SAR-Improvements through frequency and waveform diversity," in Proc. IEEE IGARSS, Boston, MA, USA, 2008, pp. V-17-V-20.

[24] F. Bordoni, M. Younis, E. Makhoul Varona, N. Gebert, and G. Krieger, "Performance investigation on scan-on-receive and adaptive digital beam-forming for high-resolution wide-swath synthetic aperture radar," in Proc. Int. ITG Workshop Smart Antennas, Berlin, Germany, 2009, pp. 114-121.

[25] F. Feng, S. Li, W. Yu, P. Huang, and W. Xu, "Echo separation in multidimensional waveform encoding SAR remote sensing using an advanced null-steering beamformer," IEEE Trans. Geosci. Remote Sens., vol. 50, no. 10 , pp. 4157-4172, Oct. 2012.

[26] R. Bamler, "A comparison of range-Doppler and wavenumber domain SAR focusing algorithms," IEEE Trans. Geosci. Remote Sens., vol. 30, no. 4, pp. 706-713, Jul. 1992.

[27] D. Garmatyuk and M. Brenneman, "Adaptive multicarrier OFDM SAR signal processing," IEEE Trans. Geosci. Remote Sens. Lett., vol. 49, no. 10 , pp. 3780-3790, Oct. 2011

[28] D. Garmatyuk, "Cross-range SAR reconstruction with multicarrier OFDM signals," IEEE Geosci. Remote Sens. Lett., vol. 9, no. 5, pp. 808812, Sep. 2012.

[29] J. Kim, M. Younis, A. Moreira, and W. Wiesbeck, "A novel OFDM chirp waveform scheme for use of multiple transmitters in SAR," IEEE Geosci. Remote Sens. Lett., vol. 10, no. 3, pp. 568-572, May 2013.

[30] C. Zetzsche and G. Krieger, "Intrinsic dimensionality: Nonlinear image operators and higher-order statistics," in Nonlinear Image Processing, S. Mitra and G. Sicuranza, Eds. New York, NY, USA: Academic, 2000, pp. 403-448.

[31] G. Krieger, I. Rentschler, G. Hauske, K. Schill, and C. Zetzsche, "Object and scene analysis by saccadic eye-movements: An investigation with higher-order statistics," Spatial Vision, vol. 13, no. 2/3, pp. 201-214, 2000.

[32] C. Sturm and W. Wiesbeck, "Waveform design and signal processing aspects for fusion of wireless communications and radar sensing," Proc. IEEE, vol. 99, no. 7, pp. 1236-1259, Jul. 2011.

[33] J. Kim, "Multiple-input multiple-output synthetic aperture radar for multimodal operation," M.S. thesis, Karlsruhe Inst. Technol. (KIT), Karlsruhe, Germany, 2012.

[34] G. Krieger, A. Moreira, H. Fiedler, I. Hajnsek, M. Werner, M. Younis, and M. Zink, "TanDEM-X: A satellite formation for high-resolution SAR interferometry," IEEE Trans. Geosci. Remote Sens., vol. 45, no. 11, pp. 3317-3341, Nov. 2007.

[35] J. H. Blythe, "Radar Systems," U.S. Patent 4253 098, Feb. 24, 1981.

[36] M. Suess and W. Wiesbeck, "Side Looking SAR System," U.S. Patent 6870 500, Mar 22, 2005.

[37] M. Suess, B. Grafmueller, and R. Zahn, "A novel high resolution, wide swath SAR system," in Proc. IEEE IGARSS, 2001, pp. 1013-1015.

[38] M. Younis, C. Fischer, and W. Wiesbeck, "Digital beamforming in SAR systems," IEEE Trans. Geosci. Remote Sens., vol. 41, no. 7, pp. 17351739, Jul. 2003.

[39] C. Fischer, C. Heer, and R. Werninghaus, "Development of a highresolution wide-swath SAR demonstrator," in Proc. EUSAR, Aachen, Germany, 2010, pp. 1-4.
[40] J. T. Kare, "Moving Receive Beam Method and Apparatus for Synthetic Aperture Radar," U.S. Patent 6175 326, Jan. 16, 2001.

[41] G. Krieger, N. Gebert, M. Younis, F. Bordoni, A. Patyuchenko, and A. Moreira, "Advanced concepts for ultra-wide-swath SAR imaging," in Proc. EUSAR, Friedrichshafen, Germany, 2008, pp. 1-4.

[42] A. Freeman, G. Krieger, P. Rosen, M. Younis, W. T. K. Johnson, S. Huber, R. Jordan, and A. Moreira, "SweepSAR: Beam-forming on receive using a reflector-phased array feed combination for spaceborne SAR," in Proc. IEEE Radar Conf., Pasadena, CA, USA, 2009, pp. 1-9.

[43] M. Younis, S. Huber, A. Patyuchenko, F. Bordoni, and G. Krieger, "Performance comparison of reflector- and planar-antenna based digital beamforming SAR," Int. J. Antennas Propag., vol. 2009, p. 614 931, 2009.

[44] S. Huber, M. Younis, G. Krieger, A. Patyuchenko, and A. Moreira, "Spaceborne reflector SAR systems with digital beamforming," IEEE Trans. Aerosp. Electron. Syst., vol. 48, no. 4, pp. 3473-3493, Oct. 2012.

[45] K.-H. Bethke, S. Baumgartner, M. Gabele, D. Hounam, E. Kemptner, D. Klement, G. Krieger, and R. Erxleben, "Air- and spaceborne monitoring of road traffic using SAR moving target indicationProject TRAMRAD," ISPRS J. Photogramm. Remote Sens., vol. 61, no. 3/4, pp. 243-259, Dec. 2006.

[46] G. Krieger, N. Gebert, M. Younis, and A. Moreira, "Advanced synthetic aperture radar based on digital beamforming and waveform diversity," in Proc. IEEE RadarCon, Rome, Italy, 2008, pp. 1-6.

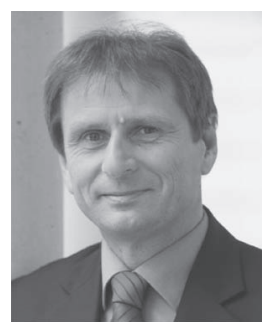

Gerhard Krieger (M'04-S'09-F'13) received the Dipl.-Ing. (M.S.) and Dr.-Ing. (Ph.D.) degrees (with honors) in electrical and communication engineering from the Technical University of Munich, Munich, Germany, in 1992 and 1999, respectively.

From 1992 to 1999, he was with Ludwig Maximilians University, Munich, Germany, where he conducted multidisciplinary research on neuronal modeling and nonlinear information processing in biological and technical vision systems. Since 1999, he has been with the Microwaves and Radar Institute (HR), German Aerospace Center (DLR), Weßling, Germany, where he developed signal and image processing algorithms for a novel forwardlooking radar system employing digital beamforming on receive. From 2001 to 2007, he led the New Synthetic Aperture Radar (SAR) Missions Group which pioneered the development of advanced bistatic and multistatic radar systems as exemplified by the TanDEM-X mission, as well as innovative multichannel SAR techniques and algorithms for high-resolution wide-swath SAR imaging. Since 2008, he has been the Head of the new Radar Concepts Department, Microwaves and Radar Institute, DLR. He has authored more than 50 peerreviewed journal papers, 7 invited book chapters, about 300 conference papers, and 5 patent families. His current research interests focus on the development of multichannel radar techniques and algorithms for innovative multiple-input multiple-output SAR systems, the demonstration of novel interferometric and tomographic Earth observation applications, and the conceptual design of advanced bi- and multistatic radar missions.

Dr. Krieger received several national and international awards, including the W.R.G. Baker Prize Paper Award from the IEEE Board of Directors and the Transactions Prize Paper Award of the IEEE Geoscience and Remote Sensing Society. In 2012, he and his colleagues were nominated for the German President's Award for Technology and Innovation. Since 2012, he has been an Associate Editor of the IEEE TRAnsACTIONS ON GEOSCIENCE AND REMOTE SENSING. 